\title{
Kinematics and vorticity in Kangmar Dome, southern Tibet: Testing midcrustal channel flow models for the Himalaya
}

\author{
Tom Wagner, ${ }^{1,2}$ Jeffrey Lee, ${ }^{1}$ Bradley R. Hacker, ${ }^{3}$ and Gareth Seward ${ }^{3}$ \\ Received 20 May 2010; revised 16 August 2010; accepted 1 September 2010; published 16 December 2010.
}

[1] Kinematic, kinematic vorticity $\left(W_{\mathrm{m}}\right)$, and deformation temperature analyses were completed to test the hypothesis that midcrustal rocks exposed in the core of the Kangmar gneiss dome, southern Tibet record ductile deformation patterns of a "frozen" segment of a southward flowing midcrustal channel. Microscopic and mesoscopic kinematic indicators exhibit a downward transition from a subequal mix of top-north and top-south shear in garnet zone rocks to dominantly top-north shear in staurolite/kyanite zone and deeper rocks. Kinematic vorticity values indicate an increase in pure shear component with depth from $\sim 48 \%$ pure shear in chloritoid zone rocks through $\sim 62 \%$ in garnet zone to staurolite/kyanite zone rocks to $\sim 68 \%$ pure shear in an orthogneiss, the deepest exposed rocks. Deformation temperatures inferred from grain-scale microstructures and quartz lattice preferred orientations increase from $\sim 300^{\circ} \mathrm{C}-400^{\circ} \mathrm{C}$ in chloritoid zone rocks to $\geq 600^{\circ} \mathrm{C}$ in the deepest exposed rocks. These temperatures are equivalent to temperatures derived from garnet-biotite thermobarometry, indicating that $W_{\mathrm{m}}$ was recorded during peak metamorphism. This ductile deformation zone was cut by the brittle southern Tibetan detachment system (STDS) that juxtaposed metasedimentary rocks upon the orthogneiss. On the basis of these relations, midcrustal rocks in the core of Kangmar Dome record: (1) general shear (vertical thinning and $\mathrm{N}-\mathrm{S}$ horizontal extension) with a component of topnorth shear during peak metamorphism within a ductile shear zone corresponding to the northern and deeper portion of the STDS, (2) an increase in pure shear with structural depth, a consequence of an increase in lithostatic load, and (3) displacement of the high-temperature shear zone by the brittle STDS. Our data are compatible with the deformation patterns predicted for the top part of a southward flowing midcrustal channel. Citation: Wagner, T., J. Lee, B. R. Hacker, and G. Seward (2010), Kinematics and vorticity in Kangmar

\footnotetext{
${ }^{1}$ Department of Geological Sciences, Central Washington University, Ellensburg, Washington, USA.

${ }^{2}$ Now at Cabot Oil and Gas, Pittsburgh, Pennsylvania, USA.

${ }^{3}$ Department of Earth Science, University of California, Santa Barbara, California, USA.

Copyright 2010 by the American Geophysical Union. 0278-7407/10/2010TC002746
}

Dome, southern Tibet: Testing midcrustal channel flow models for the Himalaya, Tectonics, 29, TC6011, doi:10.1029/2010TC002746.

\section{Introduction}

[2] The N-S collision between the Indian and Asian plates since the Eocene has resulted in impressive crustal shortening and vertical thickening in the Himalayan orogen. Yet, normal slip, parallel to convergence, along the north dipping southern Tibetan detachment system (STDS) [e.g., Burg and Chen, 1984; Burchfiel et al., 1992] has been important within this orogenic belt since at least the early Miocene [e.g., Godin et al., 2006, and references therein], and possibly as early as the late Eocene/early Oligocene [Lee and Whitehouse, 2007] (Figures 1 and 2a). A number of mechanisms have been proposed to explain normal slip along the STDS. Early models suggested that a wedge of midcrustal material, the Greater Himalayan sequence (GHS) located in the footwall of the STDS and hanging wall of the Main Central thrust (MCT), was extruded southward by gravitational collapse in response to the extreme topographic gradient along the southern margin of the Himalayan orogen [e.g., Burchfiel and Royden, 1985; Royden and Burchfiel, 1987] (Figures 1 and 2a). Vorticity and deformation temperature data from the base of the GHS in the Sutlej Valley, NW India, led Grasemann et al. [1999] and Vannay and Grasemann [2001] to propose that both simple shear and general shear characterized flow within the extruding wedge, with simple shear deformation concentrated along the boundaries of the wedge grading through general shear to pure shear extrusion of the center of the wedge.

[3] A combination of field, microstructural, and quartz petrofabric data from the lower portion of the GHS exposed in Bhutan led Grujic et al. [1996, 2002] to postulate a model whereby a layer or channel of midcrustal material (the GHS) bounded above and below by the normal-slip STDS and thrust-slip MCT, respectively, extended northward beneath southern Tibet for at least $200 \mathrm{~km}$. In this model, midcrustal ductile channel flow was characterized by Poiseuille flow or a combination of Poiseuille and Couette flow (Figure 2b). Poiseuille (or parabolic) flow develops between stationary rigid plates in which a plate-boundary-parallel gradient in pressure produces the highest velocities in the center of the channel and decreasing velocities and opposite shear senses for the top and bottom of the channel. Poiseuille flow is characterized by high vorticity number (simple shear) at the top and bottom of the channel, and no deformation at the center of the channel. Couette (or linear) flow develops between rigid plates moving relative to one another and is characterized by a linear velocity field with the highest 


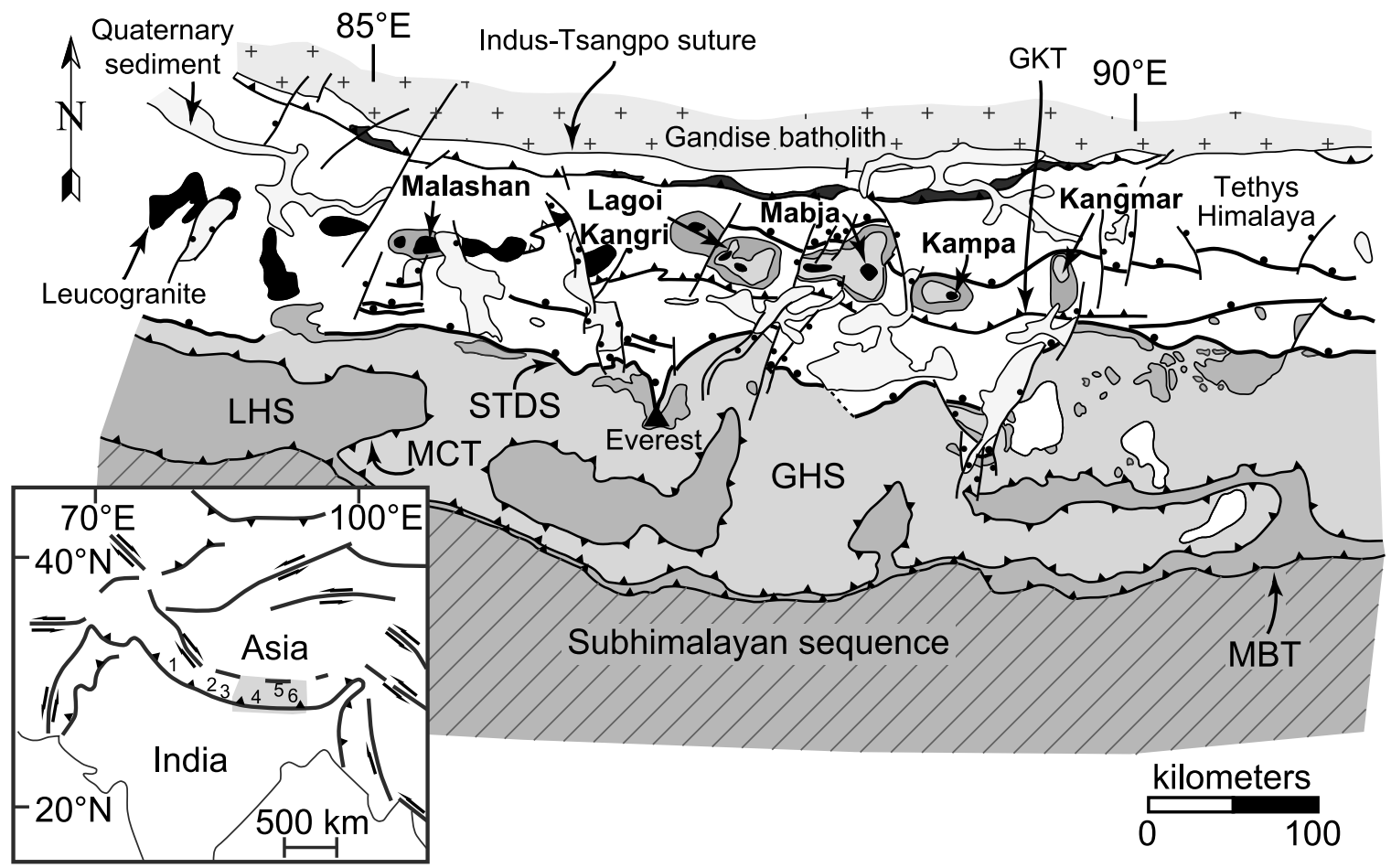

Figure 1. Regional tectonic map of the south central portion of the Himalayan orogen. Location of the north Himalayan gneiss domes (bold text), including Kangmar Dome, are shown with respect to the Greater Himalayan Sequence (GHS), Lesser Himalayan Sequence (LHS), Main Boundary Thrust (MBT), Main Central Thrust (MCT), southern Tibetan detachment system (STDS), and GyirongKangmar Thrust (GKT). Inset shows location of regional tectonic map (gray box) and numbers show locations of published vorticity studies: (1) Grasemann et al. [1999], (2) Carosi et al. [2007], (3) Larson and Godin [2009], (4) Law et al. [2004] and Jessup et al. [2006], (5) Langille et al. [2010], and (6) Carosi et al. [2006]. Modified from Lee et al. [2004].

velocities toward the top or bottom of the channel, and simple shear (high vorticity number) across the channel [e.g., White, 1974] (Figure 2b).

[4] Building on these ideas, and incorporating a wealth of geophysical and geological data from the Himalayan orogeny, Beaumont et al. [2001, 2004, 2006] developed a set of transient, plane strain, coupled thermal-mechanical finite element models in which the GHS represents a hot, lowviscosity midcrustal channel that extruded southward from beneath southern Tibet toward the orogenic front during N-S convergence (Figures $2 \mathrm{a}$ and $2 \mathrm{c}$ ). Flow began after the crust had been tectonically thickened and the midcrust had experienced a reduction in viscosity due radiogenic heating or an increase in mantle heat flux. In this model, flow and extrusion of the low-viscosity tabular body of midcrust is driven by a subhorizontal gravitational potential energy gradient developed as a consequence of the topographic and crustal thickness differences between the Tibetan Plateau and its margins, and focused erosion along the southern flank of the high Himalaya [e.g., Beaumont et al., 2001, 2004; Hodges et al., 2001]. The low-viscosity channel is bounded above and below by normal-sense (STDS) and thrust-sense (MCT) shear zones, respectively, that separate the channel from more viscous material above and below [Beaumont et al., 2001, 2004]. This model predicts that both a plate-boundary-parallel pressure gradient and underthrusting will lead to a combination of Poiseuille and Couette flow, or hybrid flow, within the channel (Figure 2b).

[5] To test the predictions of these models, kinematic, vorticity, and deformation temperature studies of the GHS were completed in the Sutlej Valley [Grasemann et al., 1999], on the Everest massif [Law et al., 2004; Jessup et al., 2006], in Bhutan [Carosi et al., 2006, 2007], and in Nepal [Larson and Godin, 2009] (Figure 1). The results indicate a deformation pattern characterized by top-north simple shear at the top, just below the STDS, an increasing component of pure shear with depth, and dominantly topsouth general shear toward the base above the MCT, a pattern consistent with that predicted by channel flow models (Figure 3a). However, Law et al. [2004] and Jessup et al. [2006] noted that the midcrustal rocks exposed on Everest might record multiple stages of a ductile deformation history that were subsequently structurally juxtaposed.

[6] Investigations similar in scale and scope to those on the Everest massif were completed on midcrustal rocks exposed in Mabja Dome, one of the North Himalayan gneiss domes (Figure 1) [Langille et al., 2010]. Midcrustal rocks exposed in the core of these domes are proposed to represent exhumed portions of the GHS [e.g., Searle et al., 2003; Beaumont et al., 2004; Lee et al., 2006; Lee and Whitehouse, 
a

South

North

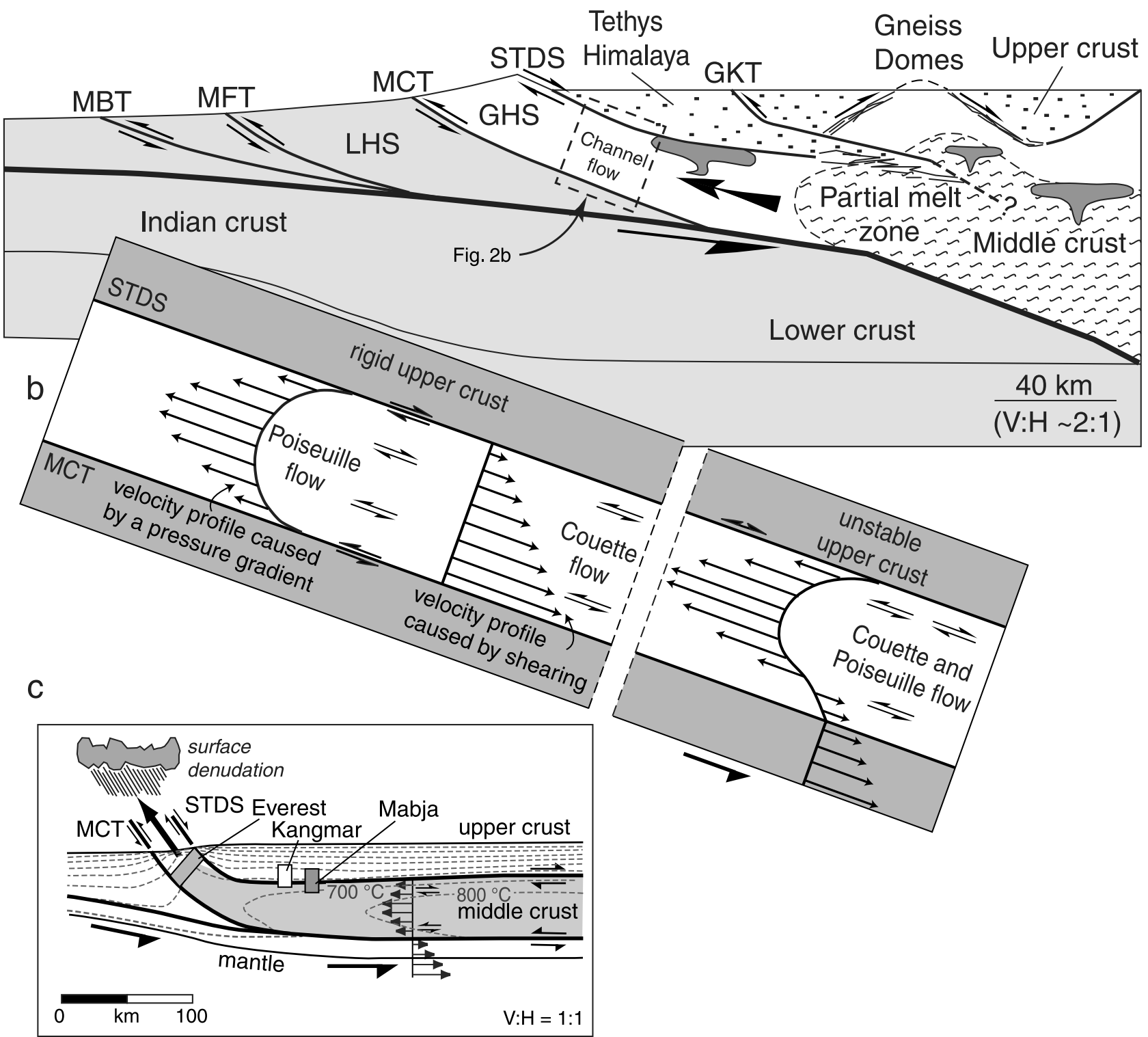

Figure 2. (a) Cross-sectional view of the central Himalayan orogeny showing major tectonic features. GHS, Greater Himalayan Sequence; GKT, Gyirong-Kangmar Thrust; LHS, Lesser Himalayan Sequence; MBT, Main Boundary Thrust; MCT, Main Central Thrust; MFT, Main Frontal Thrust; STDS, southern Tibetan detachment system. Dashed box shows location of Figure 2b. After Lee et al. [2000] and Beaumont et al. [2001]. (b) Schematic diagram showing Poiseuille, Couette, and combined CouettePoiseuille flow (hybrid flow). Predicted velocity profiles (double-barbed black arrows) and kinematics (paired single-barbed arrows). After Grujic et al. [2002], Beaumont et al. [2004], and Langille et al. [2010]. (c) Schematic cross section showing channel flow modeled isotherms and proposed locations of the Greater Himalayan Sequence (GHS) exposed on Mount Everest and middle crustal rocks exposed in the core of Mabja and Kangmar domes prior to exhumation. Paired single-barbed arrows indicate relative sense of motion across faults and sense of shear; double-barbed arrows show velocity profile; large bold arrows indicate direction of flow and exhumation of the GHS. After Beaumont et al. [2004], Godin et al. [2006], and Langille et al. [2010]. 

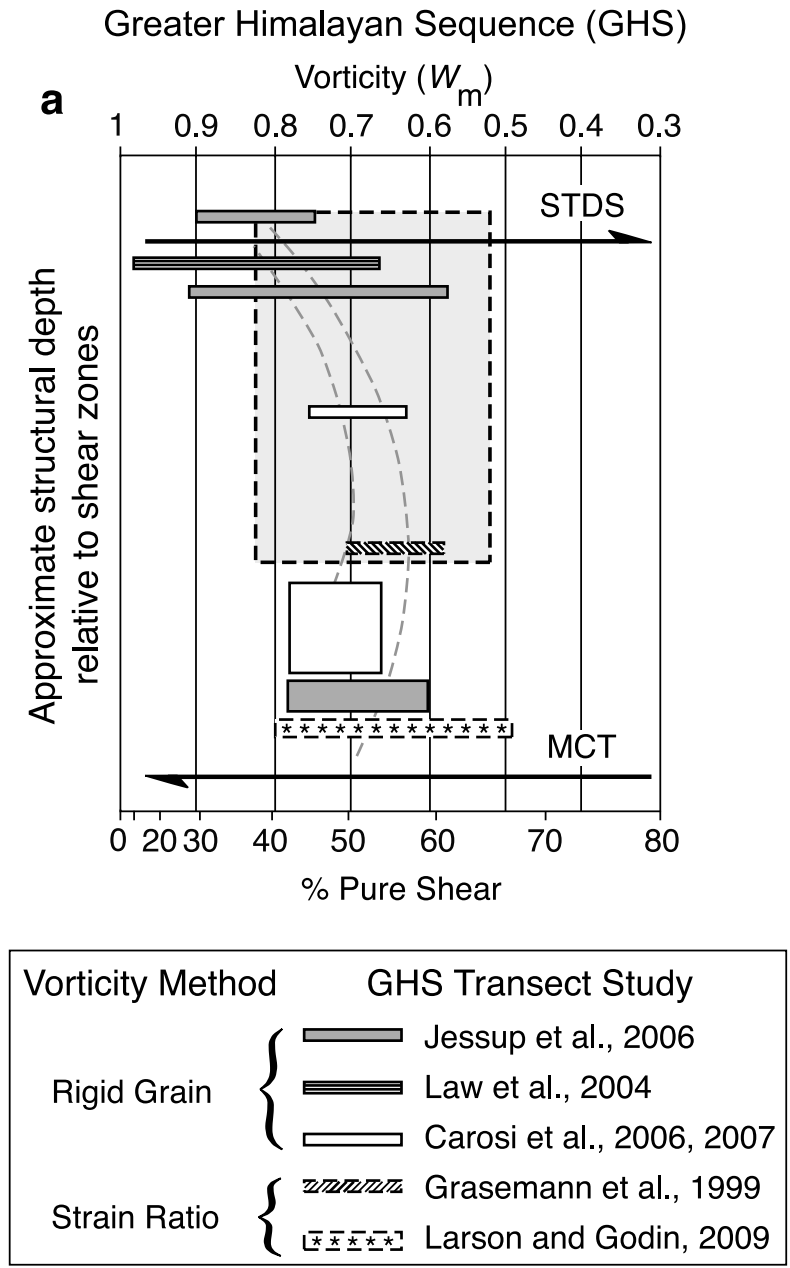
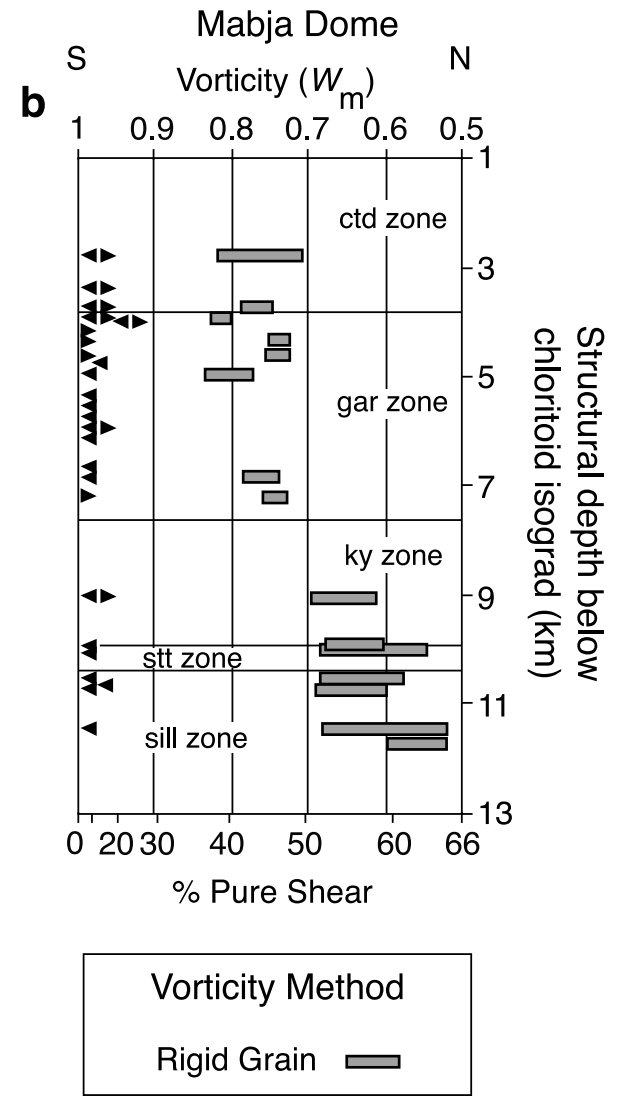

Shear sense indicators

Figure 3. Graphs of vorticity estimates versus structural depth for (a) the GHS and (b) Mabja Dome. Vorticity data from the GHS are plotted relative to the Main Central Thrust (MCT) and southern Tibetan detachment system (STDS) and from Mabja Dome are plotted with reference to structural depth below the chloritoid-in isograd (see Figure 1 for study locations). Single-barbed arrows show relative sense of motion across shear zones. Large, dashed gray rectangle in Figure 3a indicates the range in vorticity and approximate relative structural position of middle crustal rocks exposed in Mabja Dome. Abbreviations are ctd, chloritoid; gar, garnet; ky, kyanite; N, north; S, south; stt, staurolite; sill, sillimanite. After Larson and Godin [2009] and Langille et al. [2010].

2007; Cottle et al., 2009] and are closer to the presumed source of the flowing channel. Midcrustal rocks from Mabja Dome record a mix of top-north and top-south shear and $\sim 40-50 \%$ pure shear at the highest structural levels, changing with structural depth to solely top-south shear and $50-65 \%$ pure shear at the deepest structural levels (Figure 3b) [Langille et al., 2010]. Langille et al. [2010] interpreted this pattern of ductile deformation as the result of a hybrid flow regime, defined by a combination of Poiseuille and Couette flow, and flow reversal.

[7] To further test the channel flow model and to document the spatial distribution of ductile deformation patterns across southern Tibet, we report results from detailed investigations of the spatial and temporal distribution of kinematics, deformation temperatures, and vorticity in midcrustal rocks exposed in the core of Kangmar Dome, located $\sim 150 \mathrm{~km}$ east of Mabja Dome and $\sim 75 \mathrm{~km}$ north of the high Himalaya (Figure 1). Kangmar Dome exposes midcrustal rocks from $\sim 15$ to $25 \mathrm{~km}$ depth for which the structural, metamorphic, and geochronologic evolution have been well documented [Burg et al., 1984; Chen et al., 1990; Lee et al., 2000, 2002]. Our results show that ductile deformation in these midcrustal rocks was characterized by general shear: combined pure shear (vertical thinning and north-south horizontal extension) and top-north simple shear. This pattern of ductile deformation likely occurred within a ductile shear zone that was the northern and deeper continuation of the brittle normal-slip STDS. This shear zone was subsequently cut by the brittle STDS. Our results, combined with similar data sets from the GHS and from 
midcrustal rocks exposed in Mabja Dome, are consistent with models of southward ductile flow and extrusion.

\section{Geologic Setting}

\subsection{Regional Geology}

[8] Kangmar Dome is one of several gneiss domes, collectively referred to as the North Himalayan gneiss domes, exposed within the Tethys Himalaya about halfway between the Indus-Tsangpo suture and the high Himalaya (Figure 1). The Tethys Himalaya comprise a stratigraphic succession of Cambrian through Eocene marine sediments once located along the northern passive margin of India and now deformed between the Indian and Eurasian tectonic plates since collision in the Eocene [e.g., Gaetani and Garzanti, 1991; Brookfield, 1993; Liu and Einsele, 1994; Garzanti, 1999]. Exposed to the south of the North Himalayan gneiss domes is the north dipping Gyirong-Kangmar thrust fault (GKT), which juxtaposes weakly metamorphosed and deformed Tethyan sediments in its hanging wall upon unmetamorphosed Tethyan sediments in its footwall [Burg and Chen, 1984; Ratschbacher et al., 1994]. In turn, the Tethys Himalaya sits in the hanging wall of the shallowly north dipping STDS. Middle Miocene to Holocene northsouth striking grabens cut these older structures [e.g., Armijo et al., 1986; Stockli et al., 2002; Dewane et al., 2006; Hager et al., 2006; Mahéo et al., 2007].

\subsection{Geology of Kangmar Dome}

[9] The North Himalayan gneiss domes, including Malashan, Lagoi-Kangri, Mabja, Kampa, and Kangmar (Figure 1), exhibit a similar geologic framework characterized by a core of orthogneisses, migmatites, leucogranites, and highgrade metamorphic rocks mantled by progressively lower grade to unmetamorphosed rocks. These rocks record two major ductile deformation events, an older $\mathrm{N}-\mathrm{S}$ shortening and vertical thickening episode and a younger $\mathrm{N}-\mathrm{S}$ extensional and vertical thinning episode, that are bracketed in age between late Eocene/early Oligocene to middle Miocene [Chen et al., 1990; Lee et al., 2000, 2004, 2006; Zhang et al., 2004; Aoya et al., 2005, 2006; Quigley et al., 2006, 2008; Kawakami et al., 2007; Lee and Whitehouse, 2007; Liao et al., 2008].

[10] Kangmar Dome exposes a core of Cambrian orthogneiss mantled by Carboniferous and Permian metapelites overlain by Triassic sedimentary rocks that were intruded by metabasite and aplite dikes (Figures 4 and 5) [Lee et al., 2000, 2002]. The contact between the orthogneiss core and overlying metapelites has been described, most recently, as a nonconformity due to lack of stratigraphic omission and absence of a break in thermochronologic ages across it [Lee et al., 2000].

[11] Ductile fabrics within the midcrustal rocks exposed in the core of Kangmar record two major penetrative deformational events upon which lower strain events were superimposed. East-west trending F1 folds and an S1 foliation indicating north-south horizontal shortening and vertical thickening characterize the oldest deformational event, D1. The second deformational event, D2, folded bedding and the S1 foliation at high structural levels and, with increasing structural depth, transposed them into parallelism with a subhorizontal mylonitic S2 foliation and associated north-south Ls2 stretching lineation. D2 deformational fabrics indicate north-south horizontal extension and vertical thinning [Lee et al., 2000]. Rotation of F1 folds and L0x1 intersection lineations into parallelism with the Ls2 stretching lineation implies an $\mathrm{X}: \mathrm{Z}$ strain ratio of at least 30:1 associated with D2 deformation [Lee et al., 2000]. Younger, lower strain events include D3 east-west trending folds of the D2 foliation and D4 doming that folds the S2 mylonitic foliation into a doubly plunging, $\mathrm{N}-\mathrm{S}$ elongated domal geometry [Lee et al., 2000] (Figures 4 and 5).

[12] Barrovian metamorphism, characterized by concentric chloritoid- through kyanite-in metamorphic isograds increases toward the core of the dome and occurred after D1 deformation and prior to or during the early stages of D2 ductile deformation. Peak temperatures and pressures increase from $\sim 445^{\circ} \mathrm{C}$ and $\sim 370 \mathrm{MPa}$ in garnet zone rocks to $\sim 625^{\circ} \mathrm{C}$ and $\sim 860 \mathrm{MPa}$ in staurolite/kyanite zone rocks [Lee et al., 2000] (Figures 4 and 5).

[13] Thermal diffusion through thickened crust, and ${ }^{40} \mathrm{Ar} /{ }^{39} \mathrm{Ar}$ and apatite fission track thermochronology indicate that peak metamorphism, vertical thinning, and horizontal extension associated with D2 ductile deformation was likely ongoing between $\sim 40$ to $20 \mathrm{Ma}$ [Lee et al., 2000] and had ceased by $\sim 15 \mathrm{Ma}$ [Lee et al., 2000]. Contours of mica ${ }^{40} \mathrm{Ar} /{ }^{39} \mathrm{~A}$ cooling ages are domed parallel to the mylonitic S2 foliation; however, low-temperature potassium feldspar ${ }^{40} \mathrm{Ar} /{ }^{39} \mathrm{~A}$ and apatite fission track cooling ages are not, indicating that doming occurred at $\sim 11 \mathrm{Ma}$ [Lee et al., 2000].

[14] Our detailed kinematic, deformation temperature, and vorticity data on midcrustal rocks in Kangmar allow a test of the channel flow hypothesis and shed additional light on the mechanism by which this gneiss dome formed.

\section{Kinematics, Deformation Temperatures, and Vorticity}

\subsection{Introduction}

[15] One hundred and eight samples from midcrustal rocks in Kangmar Dome were analyzed to document the spatial distribution of D2 ductile deformation patterns. These samples were located within the concentric chloritoidthrough kyanite-in metamorphic isograds in Carboniferous metasediments, marbles, schists, quartzites (units $\mathrm{CPs}, \mathrm{Cm}_{2}$, $\mathrm{Cs}_{2}, \mathrm{Cm}_{1}$, and $\mathrm{Cs}_{1}$ ) and the Cambrian basal orthogneiss (unit $\mathrm{Cog}$ ) (Figures 4 and 5). Textural analyses of all 108 samples yielded information about deformation temperatures; 58 of these samples were oriented for documenting the kinematics of deformation. Vorticity analyses were completed on 36 of the 58 oriented samples to characterize the relative percentages of simple and pure shear across the dome. Electron backscatter diffraction analyses (EBSD) of nine oriented quartzite samples provide information about quartz lattice preferred orientations (LPOs) and information about temperature, shear sense, and the slip systems that were active in quartz grains during deformation. Samples mentioned by 


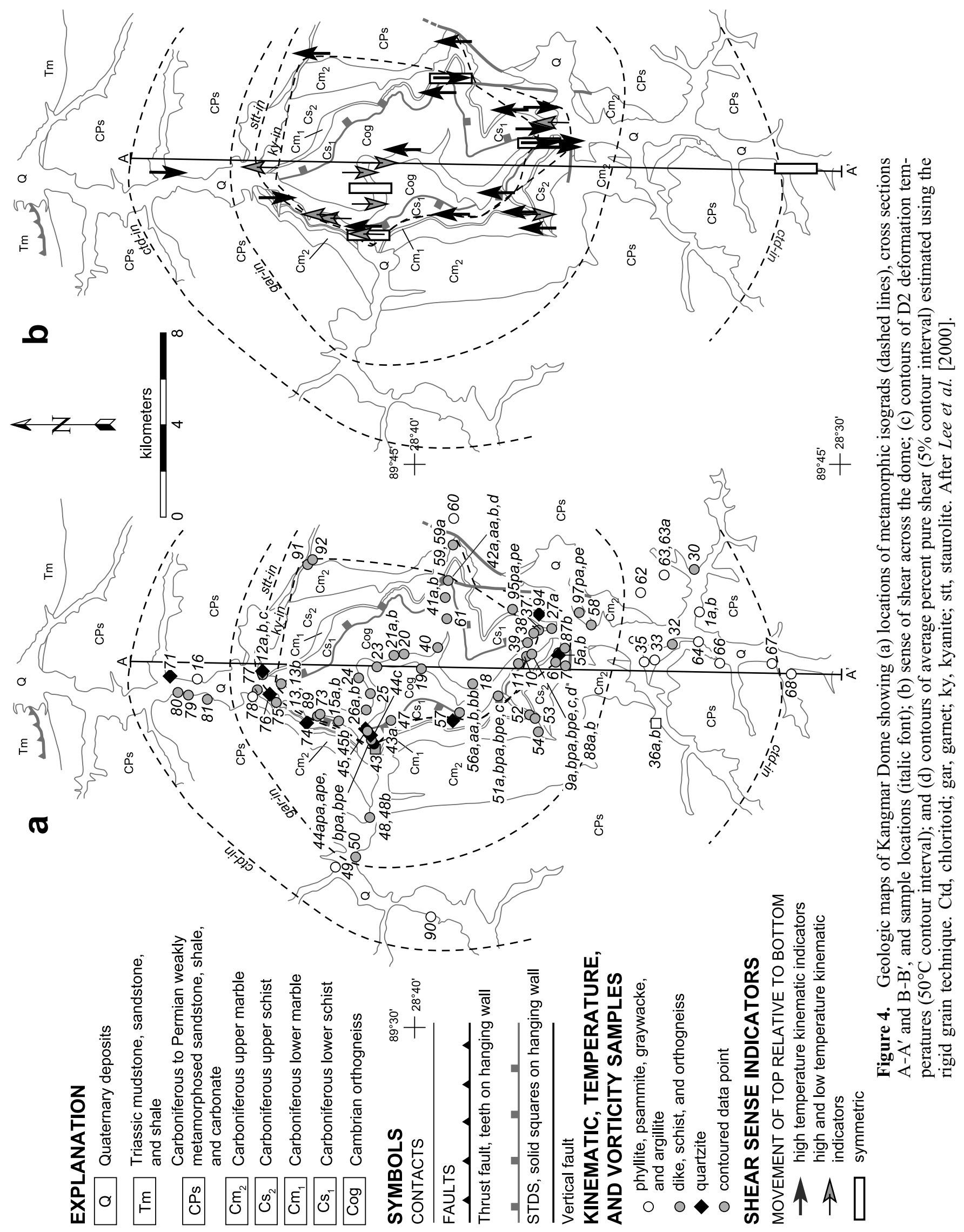




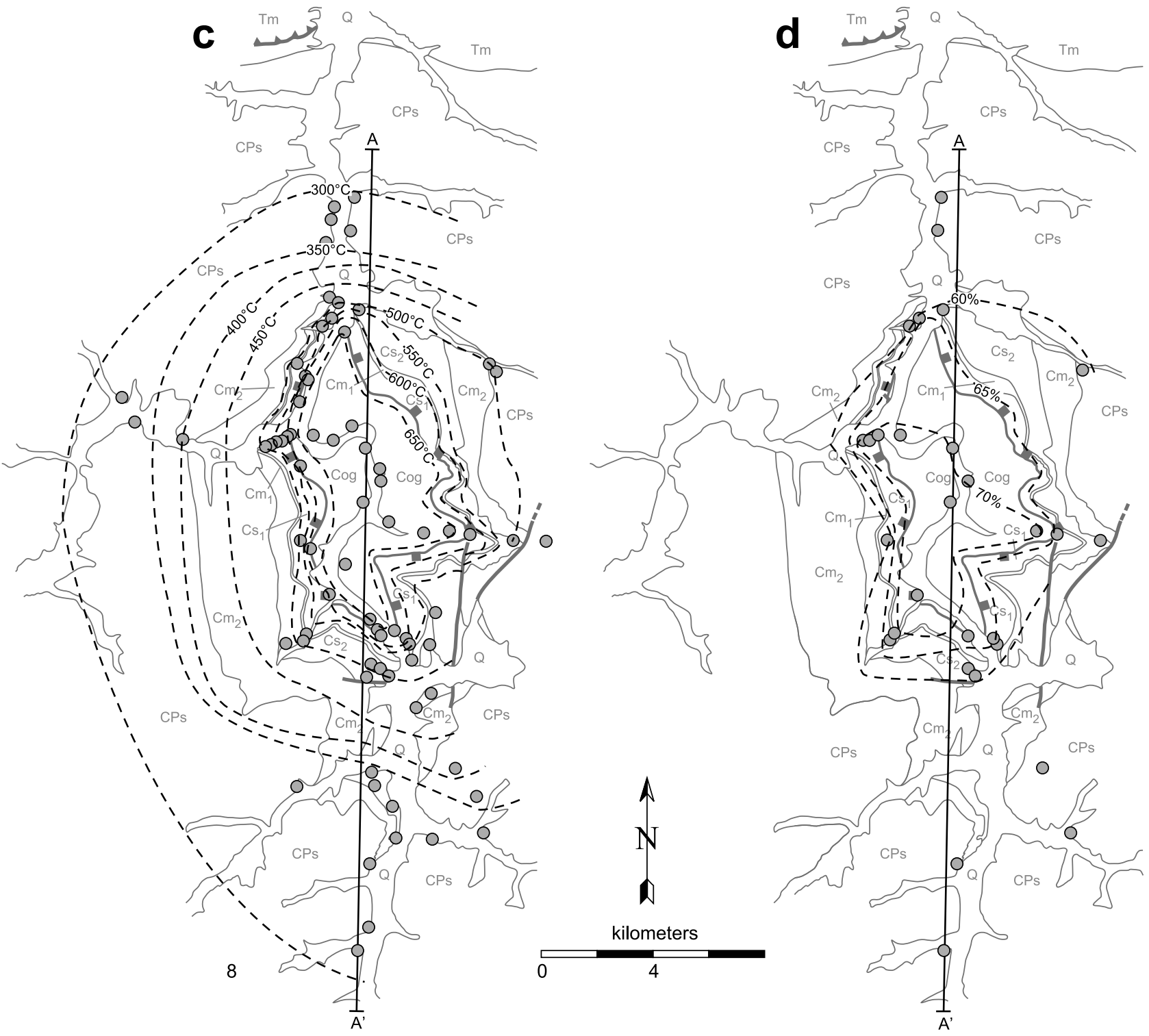

Figure 4. (continued)

name below are unique to the data set and are not representative of the whole data set.

\subsection{Kinematics}

[16] Analyses of D2 microstructures such as quartz oblique grain shape foliations (Figure 6a), asymmetric geometry of strain shadows surrounding metamorphic porphyroblasts (Figure 6b), inclusion trails of minerals within metamorphic porphyroblasts (Figure 6b), mineral fish (Figure 6c), and shear bands (Figure 6d) were completed on oriented samples to define the spatial distribution of top-north and top-south sense of shear across the dome (Figures $4 \mathrm{~b}$ and $5 b)$ (Table 1). In addition, EBSD-determined quartz [c] and (a) axis distributions were used to determine the sense of shear (Figure 7). Pfch5 software (D. Mainprice, 2005, avail- able at ftp://www.gm.univ-montp2.fr/mainprice//CareWare Unicef Programs/) was used to calculate whether [c] and $\langle\mathrm{a}\rangle$ axes defined random $(\mathrm{R})$, point $(P)$, or girdle $(G)$ distributions. The $\mathrm{R}$ value is a measure of LPO strength, where $\mathrm{R}=1$ indicates the absence of a preferred orientation. To determine strain geometry, $P$ and $G$ values were normalized, $P n$ and $G n$, respectively [Barth et al., 2010]:

$$
\begin{gathered}
P n=P /(P+G) \\
G n=G /(P+G) \text { or } G n=1-P n
\end{gathered}
$$

[17] Within chloritoid zone rocks, an oblique grain shape foliation (Figure 6a) was present in sample KD71 from the 

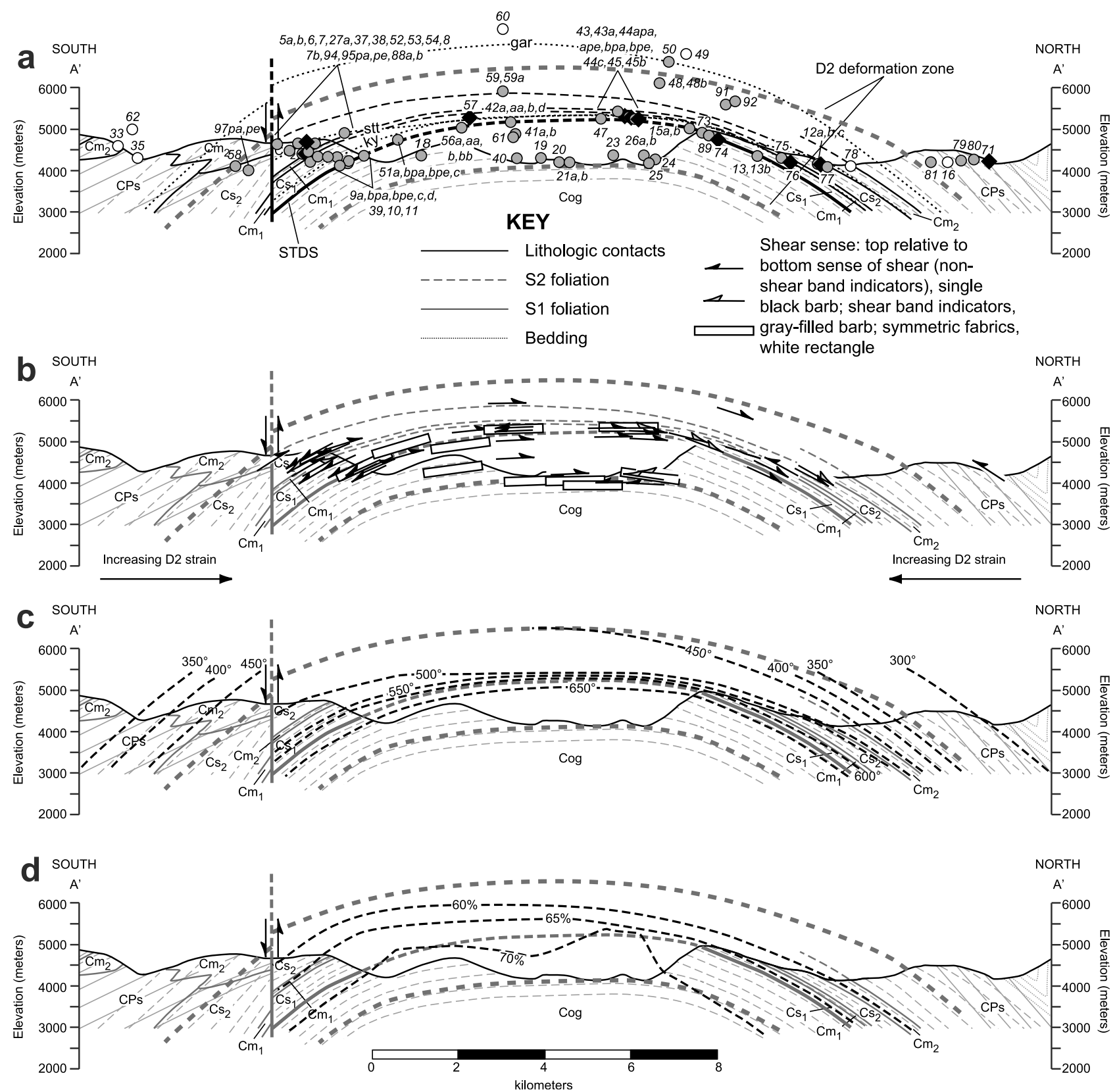

Figure 5. North-south $\left(\mathrm{A}-\mathrm{A}^{\prime}\right)$ cross sections of Kangmar Dome approximately parallel to the Ls2 stretching lineation. (a) Cross section showing geology, projected isograds and sample locations (italics), bedding, S1 and S2 foliations across the dome associated with D1 and D2 deformations, respectively, width of the D2 deformation zone (heavy dashed gray lines), and STDS (heavy solid black line). Key for sample type is given in Figure 4. (b) Cross section showing the sense of shear determined from mesoscopic and microscopic kinematic indicators across the dome. Kinematic data from our study and from Lee et al. [2000]. (c) Cross section showing deformation temperature contours. (d) Cross section showing percent pure shear deformation. Cross section modified from Lee et al. [2000].

north flank of the dome, indicating a top-south sense of shear (Figures 4b, 5b, and 8) (Table 1). Quartz LPO data from sample KD71 yielded a weak [c] axis single girdle that is oblique to the foliation by an angle of $45^{\circ}$. The distribution of the [c] and $\langle a\rangle$ axes may be the result of combined basal $\langle a\rangle$ and prism $\langle a\rangle$ slip; if so, the obliquity suggests a top-south sense of shear (Figure 7b) [cf. Barth et al., 2010]. Sample KD68 from the south flank of the dome has symmetric strain shadows surrounding chloritoid and iron oxide porphyroblasts, indicating a pure shear component (Table 1). Other samples from the chloritoid zone do not possess assessable kinematic indicators. In outcrop, scarce asymmetric 

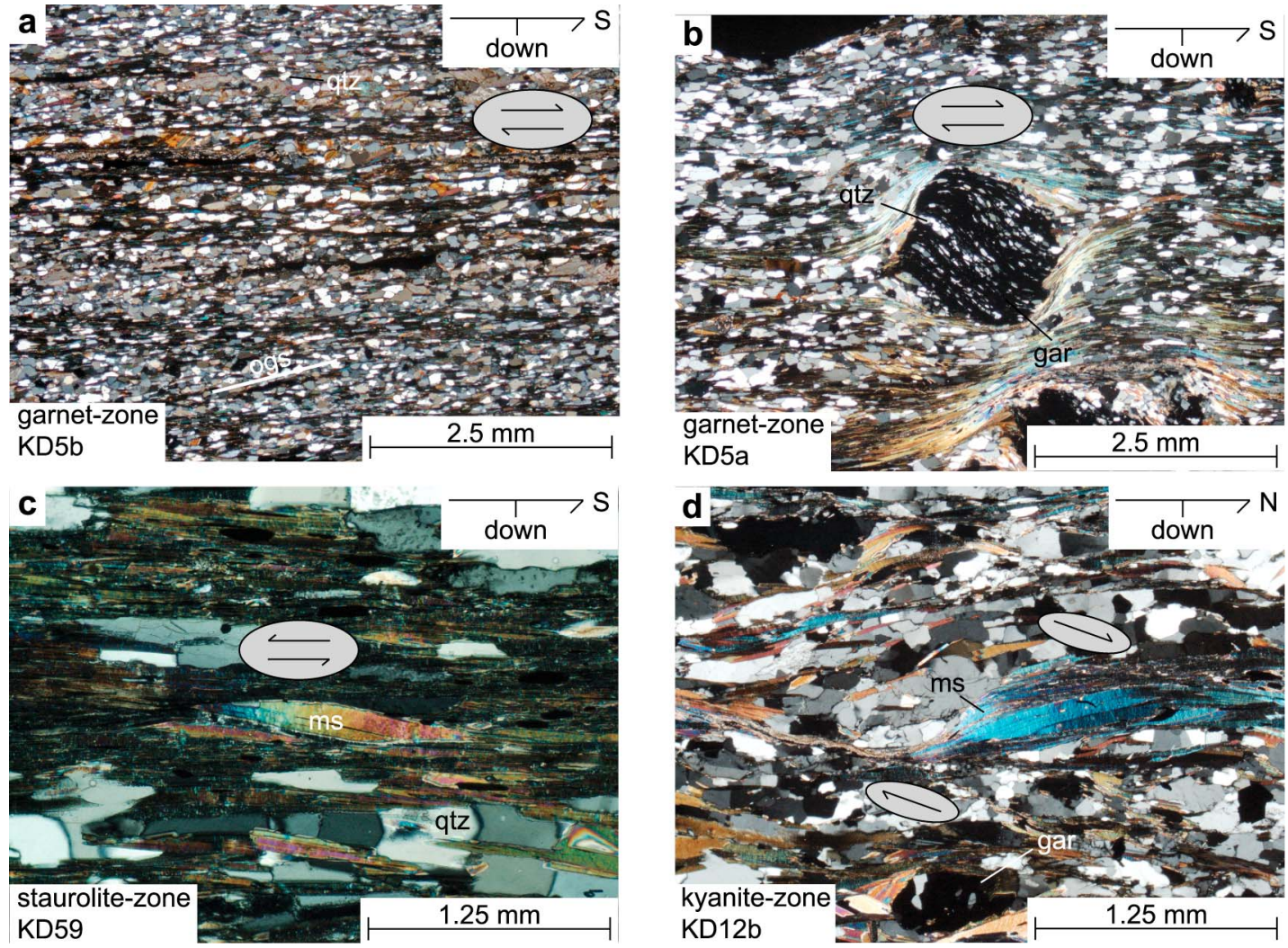

Figure 6. Photomicrographs of shear sense indicators. (a) Quartz oblique grain shape foliation indicating top-south sense of shear. (b) Asymmetric strain shadows around a garnet porphyroblast and inclusion trails of quartz indicating top-south sense of shear. (c) Mica fish indicating top-north sense of shear. (d) $\mathrm{C}^{\prime}$-type shear bands indicating top-north sense of shear. Arrow pairs indicate sense of shear, and single arrows indicate the orientation of the sample. Abbreviations: gar, garnet; ms, muscovite; qtz, quartz; N, north; S, south.

foliation boudins on the north flank of the dome suggests topnorth sense of shear.

[18] Microstructures in garnet zone rocks used to determine sense of shear include quartz oblique grain shape foliations (Figure 6a), $\sigma$ - and $\delta$-type strain shadows surrounding iron oxide and garnet porphyroblasts (Figure 6b), inclusion trails of quartz within garnet porphyroblasts (Figure 6b), and $C^{\prime}$-type shear bands (Figure 6d). Sample KD75 from the north flank of the dome and four samples from the south flank of the dome all indicate top-south sense of shear (Figures 4b, 5b, and 8) (Table 1); sample KD53 is the only one possessing microscopic shear bands, which indicate top-south sense of shear (Table 1). LPOs were measured for three quartzite samples from garnet zone rocks (Figure 7b). Samples KD5b and KD57 are both from the south flank of the dome. KD5b shows an oblique quartz grain shape foliation and a well-developed asymmetric cross-girdle [c] axis LPO (Figure 7b), both indicating a topsouth sense of shear (Table 1). Sample KD57 is characterized by a [c] axis cross-girdle LPO compatible with rhomb (a) slip; the minor asymmetry of the LPO (Figure 7) suggests top-north sense of shear (Table 1). Sample KD76, from the north flank of the dome, is characterized by a LPO compatible with basal $\langle\mathrm{a}\rangle$ slip and low $P n /$ high $G n[\mathrm{c}]$ values (Figure 7b), which suggest pure shear strain [cf. Barth et al., 2010].

[19] Kinematic microstructures observed in staurolite/ kyanite zone metapelites and down structure to the contact with the orthogneiss, include $\sigma$ - and $\delta$-type strain shadows surrounding garnet porphyroblasts, inclusion trails of quartz and mica within garnet porphyroblasts, mineral fish (Figure 6c), and microscopic $\mathrm{C}^{\prime}$-type shear bands (Figure 6d). Kinematic mesoscopic structures include shear bands, and asymmetric quartz vein and mafic dike boudins. Shear sense in these rocks is dominantly top-north across the dome (Figures $4 \mathrm{~b}$ and 5b) (Table 1). Microscopic $\mathrm{C}^{\prime}$ type shear bands are present within four of the six samples from the north flank of the dome and one sample from the south flank of the dome; all indicate top-north shear (Figures $4 \mathrm{~b}$ and $5 \mathrm{~b}$ ) (Table 1). LPO data from quartzite sample KD94 (Figure 7b), a staurolite zone rock from the south flank of the dome, yielded a well-developed asymmetric [c] axis cross-girdle and asymmetric $\langle\mathrm{a}\rangle$ axis maxima indicating a top-south sense of shear (Table 1). LPO data were collected on four quartzite samples, KD12a, 43a, 44c, 45b, from the kyanite zone along the north flank of the dome (Figure $7 \mathrm{~b}$ ). The LPO for sample KD12a has a triclinic symmetry with respect to the foliation; a sense of shear cannot be determined 
Table 1. Kangmar Dome ${ }^{\mathrm{a}}$

\begin{tabular}{|c|c|c|c|c|c|c|c|c|c|c|}
\hline Sample & $\begin{array}{l}\text { Rock } \\
\text { Type }\end{array}$ & $\begin{array}{c}\text { Microscopic } \\
\text { Shear } \\
\text { Sense }\end{array}$ & $\begin{array}{c}\text { Shear } \\
\text { Indicator }\end{array}$ & $\begin{array}{l}\text { Mesoscopic } \\
\text { Shear } \\
\text { Sense }\end{array}$ & $\begin{array}{l}\text { Shear } \\
\text { Indicator }\end{array}$ & $\begin{array}{c}\text { Matrix Deformation } \\
\text { Temperature } \\
\left({ }^{\circ} \mathrm{C}\right)\end{array}$ & $\begin{array}{l}\text { Metamorphic } \\
\text { Temperature } \\
\left({ }^{\circ} \mathrm{C}\right)\end{array}$ & $\begin{array}{l}\text { Temperature } \\
\text { Indicator }\end{array}$ & $\begin{array}{c}\text { Vorticity } \\
W_{\mathrm{m}}\end{array}$ & $\begin{array}{c}\text { Pure } \\
\text { Shear } \\
(\%)\end{array}$ \\
\hline \multicolumn{11}{|c|}{ North Flank of the Dome } \\
\hline Ctd-in & & & & & & & & & & \\
\hline $\mathrm{KD} 71^{\mathrm{b}, \mathrm{c}}$ & qtz & $\mathrm{t}-\mathrm{s}$ & ebsd/ogs & & & $\sim 400-500$ & - & quartz mstr & $0.60-0.72$ & $59-49$ \\
\hline KD80 & sch & - & - & & & $\sim 300-350$ & - & quartz mstr & - & - \\
\hline KD79 & sch & - & - & & & $\sim 300-350$ & - & quartz mstr & - & - \\
\hline $\mathrm{KD} 16^{\mathrm{c}}$ & phy & - & - & $t-n$ & $\mathrm{ab}$ & $\sim 300-350$ & - & quartz mstr & $0.78-0.89$ & $43-30$ \\
\hline KD81 & $\operatorname{sch}$ & - & - & & & $\sim 300-350$ & - & quartz mstr & - & - \\
\hline $\begin{array}{l}\text { KD49 } \\
\text { gar-in }\end{array}$ & phy & - & - & & & $\sim 300-350$ & - & quartz mstr & - & - \\
\hline KD50 & $\operatorname{sch}$ & - & - & & & - & $446 \pm 44$ & Lee et al. [2000] & - & - \\
\hline KD48 & sch & - & - & & & $\sim 400$ & - & quartz mstr & - & - \\
\hline $\mathrm{KD} 48 \mathrm{~b}$ & sch & - & - & & & $\sim 400$ & - & quartz mstr & - & - \\
\hline KD78 & psam & - & - & & & $\sim 450-550$ & - & mineral assm & - & - \\
\hline KD77 & sch & - & - & & & $\sim 450-550$ & - & mineral assm & - & - \\
\hline KD74 & qtz & - & - & & & $\sim 500-550$ & - & quartz mstr & - & - \\
\hline $\mathrm{KD} 75^{\mathrm{c}}$ & sch & $\mathrm{t}-\mathrm{s}$ & ss/it & & & $\sim 500-550$ & - & quartz mstr & $0.52-0.85$ & $65-35$ \\
\hline $\mathrm{KD}^{7} 6^{\mathrm{b}, \mathrm{c}}$ & qtz & - & - & & & $\sim 500$ & - & quartz mstr & $0.43-0.65$ & $72-54$ \\
\hline $\begin{array}{l}\text { KD43 } \\
\text { stt/ky-in }\end{array}$ & sch & - & - & & & $\sim 450-550$ & - & mineral assm & - & - \\
\hline KD92 ${ }^{\mathrm{c}}$ & sch & $t-n$ & ss & $t-n$ & mss & $\sim 500$ & - & quartz mstr & $0.49-0.59$ & $67-60$ \\
\hline KD91 & sch & - & - & & & - & $562 \pm 35$ & Lee et al. [2000] & - & - \\
\hline KD12 & & & & $t-n$ & $\mathrm{ab}, \mathrm{msb}$ & & & & & \\
\hline $\mathrm{KD} 12 \mathrm{a}^{\mathrm{c}}$ & qtz & - & - & & & $\sim 500-600$ & - & quartz mstr & $0.42-0.52$ & $72-65$ \\
\hline $\mathrm{KD} 12 \mathrm{~b}^{\mathrm{c}}$ & sch & $t-n$ & $\mathrm{ss} / \mathrm{sb}$ & & & - & $624 \pm 39$ & Lee et al. [2000] & $0.58-0.67$ & $60-53$ \\
\hline $\mathrm{KD} 12 \mathrm{c}$ & sch & - & - & & & $\sim 500-600$ & - & feldspar mstr & - & - \\
\hline KD43a $\mathrm{a}^{\mathrm{b}, \mathrm{c}}$ & qtz & - & - & & & $\sim 500$ & - & quartz mstr & - & - \\
\hline $\mathrm{KD}_{44 a p a^{\mathrm{c}}}$ & sch & sym & ss & & & $>630$ & - & quartz mstr & $0.58-0.66$ & $61-54$ \\
\hline KD44ape & sch & - & - & & & $\sim 550-600$ & - & quartz mstr & - & - \\
\hline $\mathrm{KD} 44 \mathrm{bpa}^{\mathrm{c}}$ & sch & $t-n$ & $\mathrm{sb}$ & $t-n$ & $\mathrm{ab}, \mathrm{msb}, \mathrm{mss}$ & $\sim 550-600$ & - & quartz mstr & $0.25-0.46$ & $83-70$ \\
\hline KD44bpe & sch & - & - & & & $\sim 550-600$ & - & quartz mstr & - & - \\
\hline $\mathrm{KD} 44 \mathrm{c}^{\mathrm{b}, \mathrm{c}}$ & qtz & - & - & & & $\sim 500$ & - & quartz mstr & $0.50-0.60$ & $66-59$ \\
\hline $\mathrm{KD} 45^{\mathrm{c}}$ & sch & $t-n$ & SS & $t-n$ & $\mathrm{msb}$ & $>630$ & - & quartz mstr & $0.42-0.72$ & $72-49$ \\
\hline $\mathrm{KD} 45 \mathrm{~b}^{\mathrm{c}}$ & $\mathrm{qtz}$ & - & - & & & $\sim 500$ & - & quartz mstr & $0.50-0.60$ & $66-59$ \\
\hline KD47 & sch & - & - & $t-n$ & $\mathrm{ab}, \mathrm{msb}, \mathrm{mss}$ & $\sim 500-600$ & - & quartz mstr & - & - \\
\hline $\mathrm{KD} 73^{\mathrm{c}}$ & sch & $t-n$ & $\mathrm{sb}$ & $t-n$ & mss & $\sim 500-600$ & - & feldspar mstr & - & - \\
\hline KD89 & sch & - & - & & & - & $564 \pm 38$ & Lee et al. [2000] & - & - \\
\hline $\mathrm{KD} 15 \mathrm{a}^{\mathrm{c}}$ & sch & $\mathrm{t}-\mathrm{n}$ & $\mathrm{ss} / \mathrm{sb}$ & & & $>600$ & - & feldspar mstr & - & - \\
\hline \multicolumn{11}{|l|}{ Orthogneiss } \\
\hline $\mathrm{KD} 15 \mathrm{~b}^{\mathrm{c}}$ & orth2 & - & - & & & $>630$ & - & quartz mstr & - & - \\
\hline $\mathrm{KD} 13^{\mathrm{c}}$ & orth2 & - & - & $t-n$ & $a b$ & $>630$ & - & quartz mstr & - & - \\
\hline KD13b & orth1 & - & - & & & $>630$ & - & quartz mstr & - & - \\
\hline KD14 & orth & & & $t-n$ & $\mathrm{ab}$ & & & & & \\
\hline $\mathrm{KD} 26 \mathrm{a}^{\mathrm{c}}$ & orth0 & $\mathrm{t}-\mathrm{s}$ & $\mathrm{msb} / \mathrm{maa}$ & & & $>630$ & - & quartz mstr & $0.43-0.49$ & $71-67$ \\
\hline $\mathrm{KD} 26 \mathrm{~b}$ & orth1 & - & - & & & $>630$ & - & quartz mstr & - & - \\
\hline $\mathrm{KD} 25^{\mathrm{c}}$ & meta & sym & ss & $\mathrm{t}-\mathrm{s}$ & $\mathrm{msb}$ & $\sim 500-600$ & - & quartz mstr & - & - \\
\hline $\mathrm{KD} 24^{\mathrm{c}}$ & orth1 & $\mathrm{t}-\mathrm{s}$ & $\mathrm{msb}$ & & & $>630$ & - & quartz mstr & - & - \\
\hline $\mathrm{KD} 23^{\mathrm{c}}$ & orth1 & $\mathrm{t}-\mathrm{s}$ & $\mathrm{msb}$ & & & $>630$ & - & quartz mstr & $0.41-0.51$ & $73-65$ \\
\hline KD21 & orth & & & $\mathrm{t}-\mathrm{n}, \mathrm{t}-\mathrm{s}$ & maa & & & & & \\
\hline $\mathrm{KD} 21 \mathrm{a}^{\mathrm{c}}$ & orth1 & - & - & & & $>630$ & - & quartz mstr & - & - \\
\hline $\mathrm{KD} 21 \mathrm{~b}^{\mathrm{c}}$ & apl & $t-n$ & ogs & & & $\sim 600$ & - & feldspar mstr & - & - \\
\hline $\mathrm{KD} 20^{\mathrm{c}}$ & orth0 & - & - & & & $>630$ & - & quartz mstr & $0.44-0.47$ & $70-61$ \\
\hline $\mathrm{KD} 19^{\mathrm{c}}$ & orth0 & - & - & $\mathrm{t}-\mathrm{n}, \mathrm{t}-\mathrm{s}$ & maa & $>630$ & - & quartz mstr & $0.52-0.61$ & $65-58$ \\
\hline KD46 & orth & & & $t-n$ & $\mathrm{~S} / \mathrm{C}$ & & & & & \\
\hline & & & & & South Flank o & f the Dome & & & & \\
\hline \multicolumn{11}{|l|}{ Ctd-in } \\
\hline $\mathrm{KD} 68^{\mathrm{c}}$ & phy & sym & ss & & & $\sim 300-350$ & - & quartz mstr & $0.70-0.77$ & $50-44$ \\
\hline KD67 & phy & - & - & & & $\sim 300-350$ & - & quartz mstr & - & - \\
\hline $\mathrm{KD} 66^{\mathrm{c}}$ & phy & - & - & & & $\sim 300-400$ & - & quartz mstr & $0.62-0.70$ & $57-50$ \\
\hline KD90 & phy & - & - & & & $\sim 300-400$ & - & quartz mstr & - & - \\
\hline $\mathrm{KD} 30^{\mathrm{c}}$ & sch & - & - & & & $\sim 300-350$ & - & quartz mstr & $0.67-0.80$ & $53-41$ \\
\hline KD1a & phy & - & - & & & $\sim 300-350$ & - & quartz mstr & - & - \\
\hline KD1b & phy & - & - & & & $\sim 300-350$ & - & quartz mstr & - & - \\
\hline KD64 & $\arg$ & - & - & & & $\sim 400$ & - & quartz mstr & - & - \\
\hline KD36b & sch & - & - & & & $\sim 300-350$ & - & quartz mstr & - & - \\
\hline KD33 & phy & - & - & & & $\sim 300-350$ & - & quartz mstr & - & - \\
\hline KD35 & phy & - & - & & & $\sim 300-350$ & - & quartz mstr & - & - \\
\hline $\mathrm{KD} 62^{\mathrm{c}}$ & psam & - & - & & & $\sim 400$ & - & quartz mstr & $0.70-0.80$ & $50-41$ \\
\hline
\end{tabular}


Table 1. (continued)

\begin{tabular}{|c|c|c|c|c|c|c|c|c|c|c|}
\hline Sample & $\begin{array}{l}\text { Rock } \\
\text { Type }\end{array}$ & $\begin{array}{c}\text { Microscopic } \\
\text { Shear } \\
\text { Sense }\end{array}$ & $\begin{array}{c}\text { Shear } \\
\text { Indicator }\end{array}$ & $\begin{array}{l}\text { Mesoscopic } \\
\text { Shear } \\
\text { Sense }\end{array}$ & $\begin{array}{c}\text { Shear } \\
\text { Indicator }\end{array}$ & $\begin{array}{c}\text { Matrix Deformation } \\
\text { Temperature } \\
\left({ }^{\circ} \mathrm{C}\right)\end{array}$ & $\begin{array}{l}\text { Metamorphic } \\
\text { Temperature } \\
\left({ }^{\circ} \mathrm{C}\right)\end{array}$ & $\begin{array}{l}\text { Temperature } \\
\text { Indicator }\end{array}$ & $\begin{array}{c}\text { Vorticity } \\
W_{\mathrm{m}}\end{array}$ & $\begin{array}{c}\text { Pure } \\
\text { Shear } \\
(\%)\end{array}$ \\
\hline & & & & & & & & & $0.55-0.71$ & $63-49$ \\
\hline \multicolumn{11}{|l|}{ gar-in } \\
\hline $\mathrm{KD}^{\circ} 0^{\mathrm{c}}$ & psam & - & - & & & $\sim 450-550$ & - & mineral assm & - & - \\
\hline KD58 & $\operatorname{sch}$ & - & - & & & $\sim 450-550$ & - & mineral assm & - & - \\
\hline KD97pa & sch & - & - & & & $\sim 450-550$ & - & mineral assm & - & - \\
\hline KD97pe & sch & - & - & & & $\sim 450-550$ & - & mineral assm & - & - \\
\hline KD7 & sch & - & - & & & $\sim 450-550$ & - & mineral assm & - & - \\
\hline $\mathrm{KD} 54^{\mathrm{c}}$ & sch & $t-n$ & ss/it & & & $\sim 450-550$ & - & mineral assm & - & - \\
\hline KD6 & sch & - & - & & & - & $536 \pm 39$ & Lee et al. [2000] & - & - \\
\hline $\mathrm{KD}^{2} 3^{\mathrm{c}}$ & sch & $\mathrm{t}-\mathrm{s}$ & $\mathrm{ss} / \mathrm{sb}$ & & & $\sim 500-600$ & - & feldspar mstr & $0.30-0.47$ & $80-68$ \\
\hline $\mathrm{KD} 5 \mathrm{a}^{\mathrm{c}}$ & sch & $\mathrm{t}-\mathrm{s}$ & ss/it & & & $\sim 450-550$ & - & quartz mstr & $0.56-0.62$ & $62-57$ \\
\hline \multirow[t]{2}{*}{$\mathrm{KD} \mathrm{b}^{\mathrm{b}, \mathrm{c}}$} & qtz & $\mathrm{t}-\mathrm{s}$ & ebsd/ogs & & & $\sim 450-550$ & - & opening angle & $0.60-0.72$ & $59-49$ \\
\hline & & & & & & & & & $0.93-0.99$ & $25-9$ \\
\hline $\mathrm{KD} 52^{\mathrm{c}}$ & sch & $\mathrm{t}-\mathrm{n}$ & ss/it & & & $\sim 400-500$ & - & quartz mstr & $0.43-0.60$ & $72-59$ \\
\hline $\mathrm{KD} 57^{\mathrm{b}, \mathrm{c}}$ & qtz & $t-n$ & ebsd & & & $\sim 500$ & - & quartz mstr & $0.52-0.67$ & $65-53$ \\
\hline $\begin{array}{l}\text { KD } 87 b^{c} \\
\text { stt/ky-in }\end{array}$ & sch & $\mathrm{t}-\mathrm{s}$ & $\mathrm{ss} / \mathrm{it}$ & & & $\sim 500$ & - & quartz mstr & $0.52-0.56$ & $65-62$ \\
\hline KD4 & meta & & & $\mathrm{t}-\mathrm{n}$ & $a b$ & & & & & \\
\hline KD9 & sch & & & $\mathrm{t}-\mathrm{s}$ & $\mathrm{msb}$ & & & & & \\
\hline KD9bpa ${ }^{c}$ & sch & sym & ss & & & $\sim 630$ & - & quartz mstr & $0.55-0.61$ & $62-58$ \\
\hline KD9bpe & sch & - & - & & & $\sim 630$ & - & quartz mstr & - & - \\
\hline KD9c & sch & - & - & & & $\sim 630$ & - & quartz mstr & - & - \\
\hline $\mathrm{KD} 1 \mathrm{bpa} \mathrm{c}^{\mathrm{c}}$ & sch & - & - & $\mathrm{t}-\mathrm{n}$ & $\mathrm{msb}$ & - & $554 \pm 33$ & Lee et al. [2000] & $0.41-0.53$ & $69-64$ \\
\hline KD51bpe & sch & - & - & & & - & $554 \pm 33$ & Lee et al. [2000] & - & - \\
\hline $\mathrm{KD} 51 \mathrm{c}^{\mathrm{c}}$ & sch & $t-n$ & it & $\mathrm{t}-\mathrm{n}$ & $\mathrm{msb}$ & $>600$ & - & feldspar mstr & $0.40-0.50$ & $74-66$ \\
\hline KD56b & sch & - & - & $\mathrm{t}-\mathrm{n}$ & $\mathrm{msb}$ & $>630$ & - & quartz mstr & - & - \\
\hline KD56bb & sch & - & - & & & $>630$ & - & quartz mstr & - & - \\
\hline $\mathrm{KD} 59^{\mathrm{c}}$ & sch & $t-n$ & $\mathrm{ss} / \mathrm{it} / \mathrm{mf}$ & & & $\sim 500$ & - & quartz mstr & $0.60-0.68$ & $59-52$ \\
\hline KD59a & sch & - & - & & & $\sim 500$ & - & quartz mstr & - & - \\
\hline KD94 $4^{\text {b,c }}$ & qtz & $\mathrm{t}-\mathrm{s}$ & ebsd & & & $\sim 430-530$ & - & opening angle & $0.55-0.68$ & $63-52$ \\
\hline KD95pa ${ }^{c}$ & sch & $t-n$ & ss/it & & & $\sim 500$ & - & quartz mstr & - & - \\
\hline KD95pe & sch & - & - & & & $\sim 500$ & - & quartz mstr & - & - \\
\hline $\mathrm{KD} 27 \mathrm{a}^{\mathrm{c}}$ & sch & $t-n$ & $\mathrm{sb}$ & & & $\sim 500$ & - & quartz mstr & - & - \\
\hline KD28 & qtz vein & & & $\mathrm{t}-\mathrm{s}$ & $a b$ & & & & & \\
\hline $\mathrm{KD} 37^{\mathrm{c}}$ & sch & - & - & & & $\sim 600$ & - & feldspar mstr & $0.48-0.60$ & $68-59$ \\
\hline $\mathrm{KD} 38^{\mathrm{c}}$ & sch & $\mathrm{t}-\mathrm{s}$ & ss/it & $\mathrm{t}-\mathrm{s}$ & mss & $\sim 500$ & - & quartz mstr & $0.67-0.76$ & $53-45$ \\
\hline KD42 & sch & & & $\mathrm{t}-\mathrm{n}$ & $\mathrm{msb}$ & & & & & \\
\hline $\mathrm{KD} 42 \mathrm{~b}^{\mathrm{c}}$ & sch & $\mathrm{t}-\mathrm{s}$ & $\mathrm{ss} /$ it & & & - & $614 \pm 30$ & Lee et al. [2000] & $0.56-0.65$ & $62-54$ \\
\hline $\mathrm{KD} 42 \mathrm{~d}^{\mathrm{c}}$ & sch & sym & ss & & & $>630$ & - & quartz mstr & - & - \\
\hline \multicolumn{11}{|l|}{ Orthogneiss } \\
\hline $\mathrm{KD} 42 \mathrm{a}^{\mathrm{c}}$ & orth1 & - & - & & & $>630$ & - & quartz mstr & - & - \\
\hline KD42aa & orth1 & - & - & $\mathrm{t}-\mathrm{n}$ & $\mathrm{msb}$ & $>600$ & - & feldspar mstr & - & - \\
\hline KD88a & orth3 & - & - & & & $>630$ & - & quartz mstr & - & - \\
\hline $\mathrm{KD} 88 \mathrm{~b}$ & orth0 & - & - & & & $>630$ & - & quartz mstr & - & - \\
\hline KD9 $a^{c}$ & orth3 & $\mathrm{t}-\mathrm{s}$ & ss & & & $>630$ & - & quartz mstr & - & - \\
\hline KD9d & orth1 & - & - & & & $>630$ & - & quartz mstr & - & - \\
\hline $\mathrm{KD} 51 \mathrm{a}^{\mathrm{c}}$ & orth1 & - & - & & & $\sim 550-600$ & - & quartz mstr & - & - \\
\hline $\mathrm{KD} 6 \mathrm{a}^{\mathrm{c}}$ & orth2 & - & - & & & $>630$ & - & quartz mstr & - & - \\
\hline KD56aa & orth2 & - & - & & & $>630$ & - & quartz mstr & - & - \\
\hline $\mathrm{KD} 9^{\mathrm{c}}$ & orth1 & - & - & $\mathrm{t}-\mathrm{n}$ & $\mathrm{msb}$ & $>630$ & - & quartz mstr & - & - \\
\hline $\mathrm{KD} 10^{\mathrm{c}}$ & orth2 & - & - & & & $>630$ & - & quartz mstr & - & - \\
\hline $\mathrm{KD} 11^{\mathrm{c}}$ & orth1 & - & - & $\mathrm{t}-\mathrm{s} ; \mathrm{t}-\mathrm{n} ; \mathrm{sym}$ & maa & $>630$ & - & quartz mstr & - & - \\
\hline $\mathrm{KD} 41 \mathrm{a}^{\mathrm{c}}$ & orth2 & - & - & & & $\sim 600-700$ & - & feldspar mstr & $0.38-0.55$ & $75-63$ \\
\hline $\mathrm{KD} 41 \mathrm{~b}^{\mathrm{c}}$ & orth2 & $t-n$ & mss & & & $\sim 600-700$ & - & feldspar mstr & $0.38-0.50$ & $75-66$ \\
\hline $\mathrm{KD} 18^{\mathrm{c}}$ & orth0 & - & - & & & $\sim 600-700$ & - & feldspar mstr & - & - \\
\hline KD61 & orth0 & - & - & & & $\sim 600-700$ & - & feldspar mstr & - & - \\
\hline $\mathrm{KD} 40^{\mathrm{c}}$ & orth1 & - & - & & & $\sim 600-700$ & - & feldspar mstr & - & - \\
\hline
\end{tabular}

${ }^{\mathrm{a}}$ Samples are in order of increasing structural depth from the north and south flanks of the dome. Samples with two vorticity values represent rigid grain (regular text) and oblique grain-shape foliation (italic text) techniques that were applied to a single sample. Abbreviations: ab, asymmetric boudin; apl, aplite dike; arg, argillite; assm, assemblage; ebsd, electron backscatter diffraction; it, inclusion trails; maa, mesoscopic asymmetric augen; meso, mesoscopic; micro, microscopic; meta, metabasite dike; mf, mineral fish; msb, mesoscopic shear band; mss, mesoscopic strain shadow; mstr, microstructures; ogs, oblique grain-shape foliation; orth, orthogneiss; phy, phyllite; psam, psammite; qtz, quartzite; sb, shear bands; S/C, S/C fabrics; sch, schist; ss, strain shadow; sym, symmetric; t-n, top-north; t-s, top-south. Orth0, orthogneiss sample with no foliation; orth 1 , orthogneiss sample with weakly aligned biotite; orth2, orthogneiss sample with weakly flattened quartz grains and aligned biotite; orth3, orthogneiss sample with strongly flattened quartz grains and aligned biotite.

${ }^{\mathrm{b}}$ Sample analyzed with EBSD.

${ }^{\mathrm{c}}$ Sample oriented parallel to Ls2. 

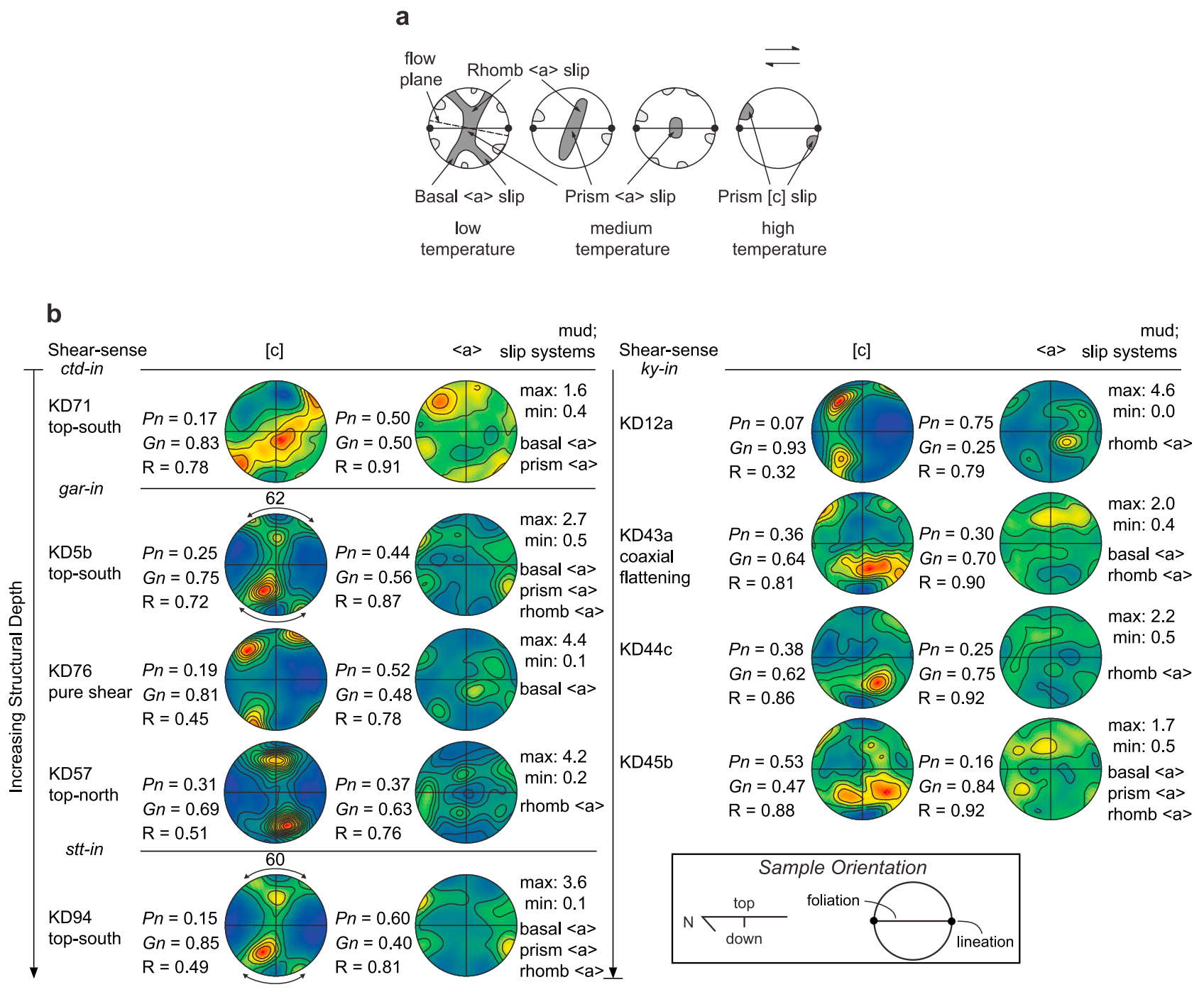

Figure 7. Common and measured quartz lattice-preferred orientation (LPO) data showing the development of $[\mathrm{c}]$ and $\langle a\rangle$ axes patterns as a function of increasing temperature. (a) Lower hemisphere pole figures showing various $[\mathrm{c}]$ and $\langle\mathrm{a}\rangle$ axis patterns commonly observed during progressive noncoaxial dextral shear where the $[\mathrm{c}]$ axes are shaded in medium gray and $\langle\mathrm{a}\rangle$ axes are shaded in light gray. After Passchier and Trouw [2005]. (b) Quartz LPO data from EBSD analyses of quartzite samples showing lower hemisphere plots of [c] and poles to (21-10) $(\langle\mathrm{a}\rangle)$ axes. Metamorphic isograds, shear sense, sample orientation, and normalized $(P n)$ and $(G n)$ values with corresponding LPO strength (R). Data are points per grain; contours are mean uniform density (mud) with maximum (max) and minimum (min) values. Pfch5 software was used to create the contoured quartz [c] and $\langle\mathrm{a}\rangle$ axis pole figures.

from this sample. Sample KD43a yields [c] and $\langle a\rangle$ axis small-circle girdles suggesting coaxial flattening (Figure $7 \mathrm{~b}$ ) [cf. Barth et al., 2010]. Samples KD44c and KD45b also did not yield interpretable LPOs, implying that the LPO and L-S fabric reflect different deformation histories.

[20] Kinematic indicators observed in orthogneiss at and below the contact with the metapelites include microscopic and mesoscopic strain shadows, quartz oblique grain shape foliations, mesoscopic asymmetric augen, sheared quartz veins, and shear bands (Table 1). These indicators yield a mix of top-north and top-south senses of shear on the north flank of the dome and dominantly top-north sense of shear on the south flank (Figures $4 \mathrm{~b}$ and $5 \mathrm{~b}$ ) (Table 1). These kinematic fabrics are best preserved within a high-strain, well-developed mylonitic foliation, defined by strongly flattened and elongate quartz grains and aligned micas, and associated with a $\sim \mathrm{N}-\mathrm{S}$ trending stretching lineation. This high-strain fabric is located within $\sim 20 \mathrm{~m}$ of the contact with the overlying metapelites. The foliation, lineation, and kinematic fabrics become progressively weaker with depth and disappear $\sim 1000 \mathrm{~m}$ below the contact between the orthogneiss and overlying metapelites. Below this boundary, the D2 
SOUTHERN HALF

$S$

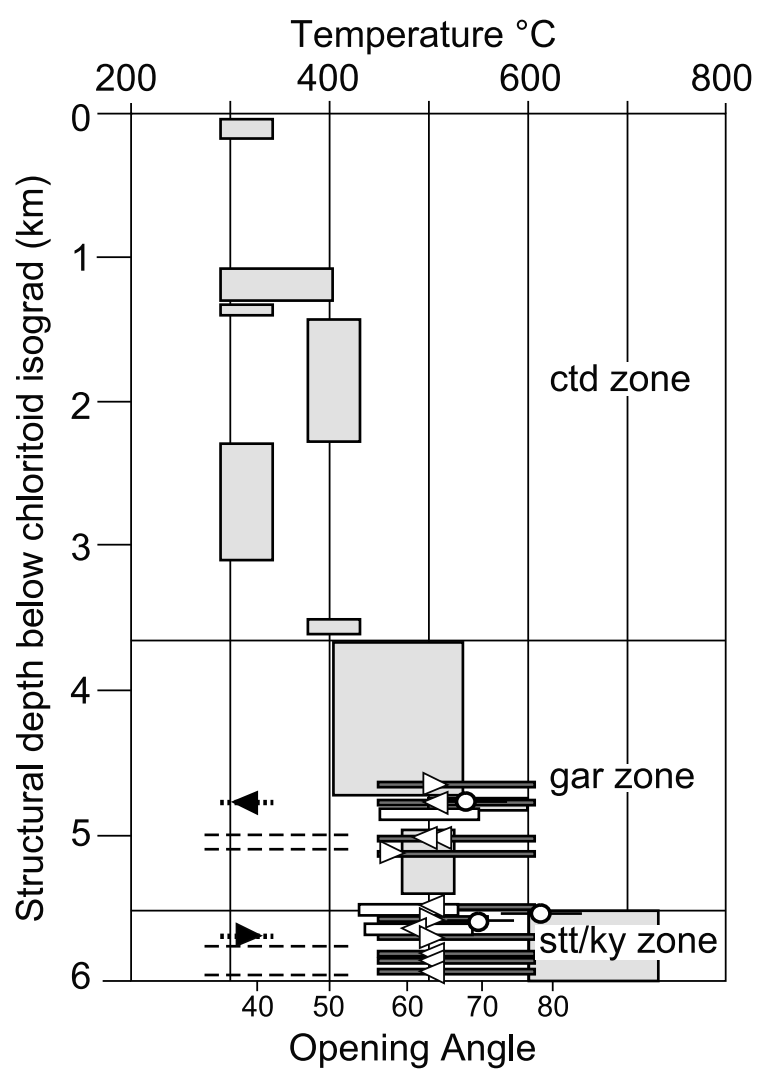

$\mathrm{N}$
NORTHERN HALF

$S$

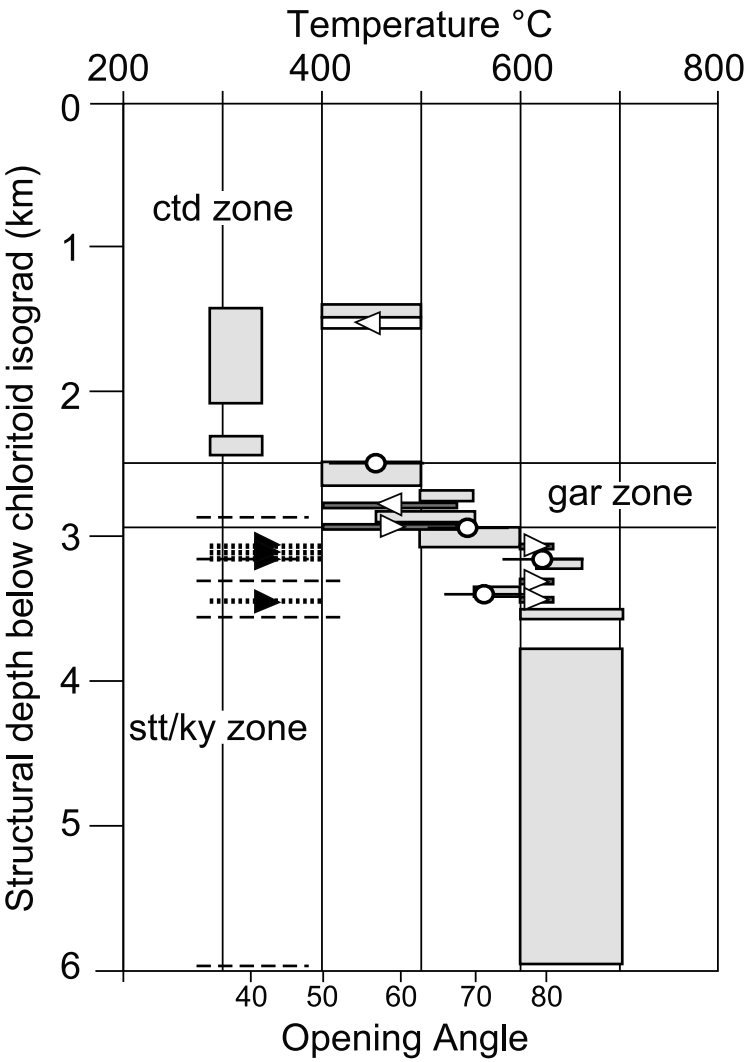

$\mathrm{N}$

800

Matrix deformation temperatures from quartz, feldspar, and calcite microstructures

Deformation temperatures from opening angles of quartz [c]-axis LPOs and quartz slip systems inferred from LPOs

Deformation temperatures from mineral assemblages in strain shadows

......... Deformation temperatures from quartz microstructures in shear bands

- - Fe-Mg exchange thermometry from Lee et al. (2000)

- - - Muscovite and biotite ${ }^{40} \mathrm{Ar} /{ }^{39} \mathrm{Ar}$ closure temperatures from Lee et al. (2000)

$\triangleright$ Sense of shear observed in strain shadows, rotated porphyroblasts, quartz LPOs, and quartz oblique grain-shape foliations

- Sense of shear observed in shear bands

Figure 8. Estimated deformation temperatures for sample matrix and kinematic indicators and sense of shear versus structural depth for schists, quartzites, and orthogneisses for the northern and southern halves of the Kangmar Dome. The width of the rectangles represents the estimated temperature range, and the height represents the range in structural depth for single and multiple samples over which those temperatures are observed. Mesoscopic shear sense indicators without deformation temperature estimates are not shown. Abbreviations are ctd, chloritoid; gar, garnet; ky, kyanite: stt, staurolite.

mylonitic foliation is not present, and the orthogneiss appears undeformed.

\subsection{Deformation Temperatures}

[21] Methods used to assess deformation temperatures recorded in midcrustal rocks in Kangmar include quartz
(Figures 9a-9e), feldspar (Figure 9f), and calcite microstructures; the stability fields of mineral assemblages present within the strain shadows surrounding chloritoid, garnet, staurolite, and kyanite porphyroblasts; and the opening angle in quartz [c] axis LPOs. For the latter, the opening angle is defined as the angle between the two girdles of [c] axis crossgirdle LPOs. A positive correlation exists between deforma- 

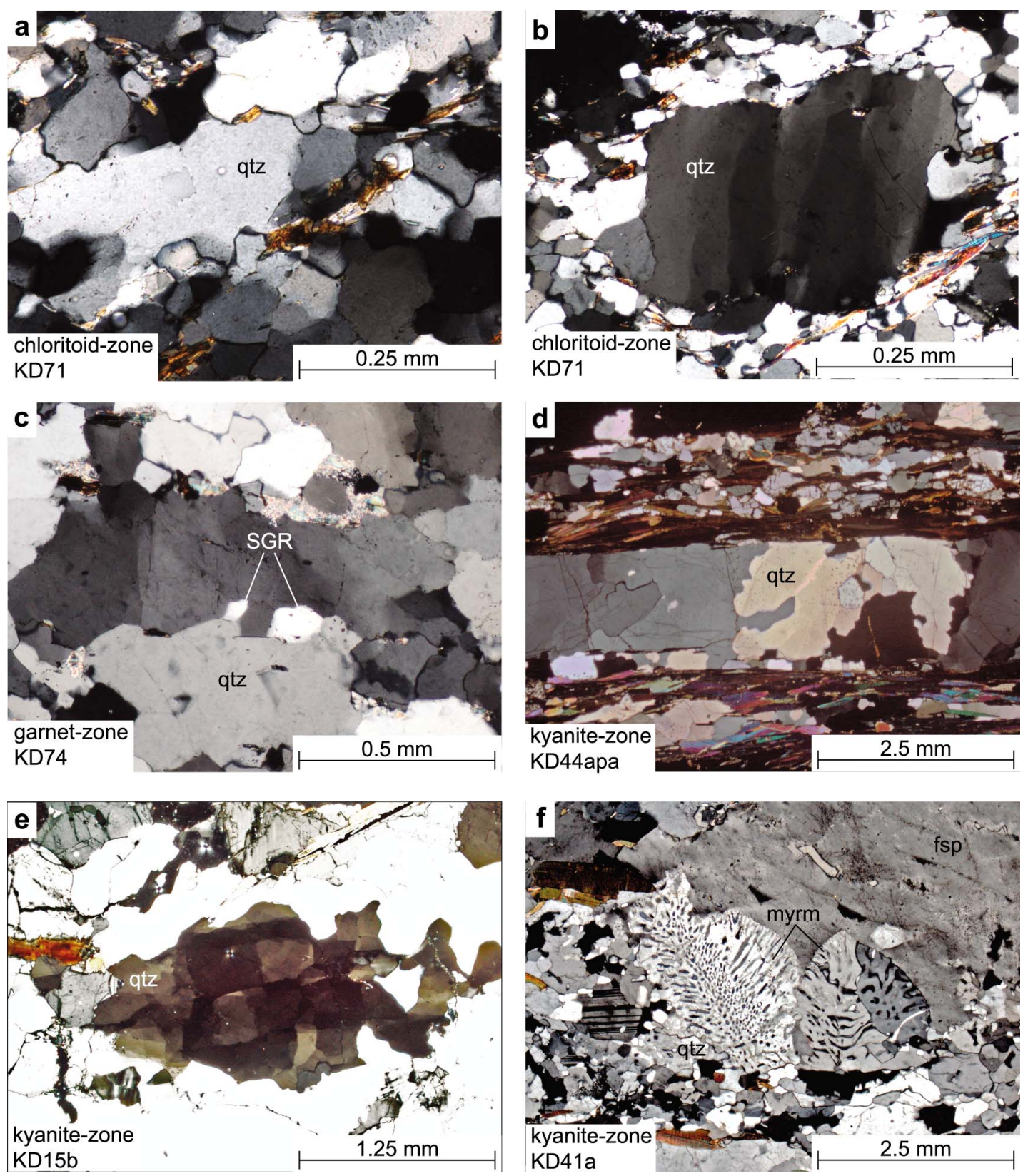

Figure 9. Photomicrographs of microstructures from which deformation temperatures were inferred. (a) Bulging recrystallization (BLG) in quartz $\left(\sim 280-400^{\circ} \mathrm{C}\right)$. (b) Undulose extinction in quartz $\left(\sim 300-350^{\circ}\right.$ C). (c) Subgrain rotation (SGR) in quartz $\left(\sim 400-500^{\circ} \mathrm{C}\right)$. (d) Grain boundary migration (GBM) in quartz $\left(\sim 500^{\circ} \mathrm{C}\right)$. (e) Chessboard extinction in quartz $\left(\geq 630^{\circ} \mathrm{C}\right)$. (f) Myrmekite in feldspar $\left(\geq 600^{\circ} \mathrm{C}\right)$. Abbreviations are gar, garnet; myrm, myrmekite; qtz, quartz.

tion temperature and opening angle between $\sim 250^{\circ} \mathrm{C}$ and $650^{\circ} \mathrm{C} \pm 50^{\circ} \mathrm{C}$, although hydrolytic weakening and changes in strain rate also play a role [Law et al., 2004]. In this study, deformation temperatures estimated from quartz [c] axis opening angles (Figure 7) overlap with deformation temperature estimates based on quartz and feldspar microstructures (Figure 8), supporting the assumption that opening angle increases with increasing temperature.

[22] Matrix quartz grains within this zone exhibit bulging recrystallization microstructures (Figure 9a) and undulatory extinction (Figure 9b), with deformation lamellae more noticeable toward the base of the chloritoid zone, indicating deformation temperatures of $\sim 300^{\circ} \mathrm{C}$ increasing to $\sim 400^{\circ} \mathrm{C}$ with structural depth [Hirth and Tullis, 1992; Stipp et al., 2002a; Passchier and Trouw, 2005] (Figure 8). Sample KD81, a calc schist located near the base of the chloritoid zone, contains strained, but untwinned, calcite grains, indicating temperatures of $>300^{\circ} \mathrm{C}$ [Burkhard, 1993; Ferrill et al., 2004]. The inference of basal $\langle\mathrm{a}\rangle$ and prism $\langle\mathrm{a}\rangle$ slip and lack of prism [c] slip from quartz LPO data within 
chloritoid zone rocks (Figure 7b) also indicate greenschist to amphibolite facies temperatures [Mainprice et al., 1986].

[23] In garnet zone rocks, deformation temperatures of $\sim 450^{\circ} \mathrm{C}-550^{\circ} \mathrm{C}$ are indicated by quartz + biotite + muscovite parageneses in strain shadows surrounding rotated garnet porphyroblasts [Spear, 1993] (Table 1). Matrix quartz grains in this zone include undulatory extinction, deformation lamellae, bulging recrystallization (Figure 9c), and subgrain rotation microstructures indicating temperatures of $\sim 300-500^{\circ} \mathrm{C}$ [Hirth and Tullis, 1992; Stipp et al., 2002a; Passchier and Trouw, 2005]. The core-and-mantle microstructure indicative of subgrain rotation in matrix quartz grains is more noticeable with increasing structural depth, indicating deformation temperatures of $\sim 400-500^{\circ} \mathrm{C}$ near the base of garnet zone rocks [Hirth and Tullis, 1992; Stipp et al., 2002a; Passchier and Trouw, 2005] (Figure 8). Two samples, KD5b and KD97pa, contain calcite grains with a few thick tabular twins; however, most grains lacked twins, suggesting temperatures of $>300^{\circ} \mathrm{C}$ [Passchier and Trouw, 2005]. Temperatures of kinematic indicators such as microscopic shear bands determined by quartz textures and strain shadows determined by mineral assemblages ranged from $\sim 280$ to $350^{\circ} \mathrm{C}$ and $\sim 400$ to $550^{\circ} \mathrm{C}$, respectively (Figure 8). Quartz LPOs from this zone show an increase with structural depth from inferred basal $\langle a\rangle$, prism $\langle a\rangle$, and rhomb $\langle a\rangle$ slip to exclusively rhomb $\langle a\rangle$ slip, indicating an increase in temperatures from $\sim 400^{\circ} \mathrm{C}$ to $<650^{\circ} \mathrm{C}$ [Mainprice et al., 1986]. Sample KD5b yields a cross-girdle [c] axis pattern and opening angle of $\sim 62^{\circ}$, corresponding to deformation temperatures of $\sim 450^{\circ} \mathrm{C}-550^{\circ} \mathrm{C}$ (Figure $7 \mathrm{~b}$ ) (Table 1).

[24] Deformation temperatures $\geq 600^{\circ} \mathrm{C}$ were recorded in the deepest exposed section of the dome by quartz + biotite + muscovite parageneses [Spear, 1993] in strain shadows that are in equilibrium with rotated kyanite porphyroblasts within staurolite/kyanite zone rocks (Figure 8). Matrix quartz grains throughout this zone exhibited microstructures indicative of grain boundary migration recrystallization (Figure 9d) and minor chessboard extinction (Figure 9e), indicating deformation temperatures of $\sim 500$ to $>630^{\circ} \mathrm{C}$, respectively [Hirth and Tullis, 1992; Stipp et al., 2002a; Passchier and Trouw, 2005]. The presence of myrmekite in matrix feldspars at the deepest structural levels indicates temperatures in excess of $\sim 600^{\circ} \mathrm{C}$ [Burkhard, 1993; Ferrill et al., 2004] (Figure 9f). Microscopic shear bands in this zone have deformation temperatures determined by quartz and feldspar microstructures of $\sim 300$ to $400^{\circ} \mathrm{C}$, and strain shadow mineral assemblages indicate $\sim 450$ to $>600^{\circ} \mathrm{C}$ (Figure 8). Quartz LPOs, except sample KD94, in this zone are triclinic with respect to the foliation and lineation (Figure 7b). Sample KD94, from the structurally highest part of this zone, yielded a cross-girdle [c] axis LPO with an opening angle of $\sim 60^{\circ}$ corresponding to a deformation temperature of $\sim 430-530^{\circ} \mathrm{C}$ (Figure $7 \mathrm{~b}$ ) (Table 1).

[25] Matrix and strain shadow deformation temperature estimates in chloritoid through kyanite zone rocks overlap with peak metamorphic temperatures estimated by Lee et al. [2000] on the basis of $\mathrm{Fe}-\mathrm{Mg}$ partitioning between biotite and garnet (Figure 8) (Table 1). These relations support Lee et al.'s [2000] interpretation that D2 deformation occurred during and after the peak metamorphism. Contours of matrix deformation temperatures (Figures $4 \mathrm{c}$ and $5 \mathrm{c}$ ) were created using the median value of the estimated range in matrix deformation temperatures for each sample (Table 1). These contours are at best approximate and depend on how each temperature estimate is weighted and whether different samples with overlapping ranges in deformation temperature are grouped together or treated separately. Regardless, D2 deformation temperature contours are domed across Kangmar (Figure $5 \mathrm{c}$ ), confirming that doming postdates D2 deformation [Lee et al., 2000].

[26] The deformation temperatures of microscopic shear bands determined by quartz and feldspar microstructures overlap with muscovite and biotite ${ }^{40} \mathrm{Ar} /{ }^{39} \mathrm{Ar}$ closure temperatures $\left(\sim 285-420^{\circ} \mathrm{C}\right)$ [Lee et al., 2000] and are cooler than matrix deformation temperatures (Figure 8). This observation, combined with the fact that the shear bands cut across the mylonitic S2 foliation and D2 ductile fabrics, requires that these fabrics formed after the $\mathrm{D} 2$ ductile deformation.

\subsection{Vorticity}

[27] Mean vorticity numbers were estimated at Kangmar using oriented thin sections cut perpendicular to the foliation and parallel to the Ls2 stretching lineation. Measurements for vorticity analyses were either taken directly from thin sections using a petrographic microscope or from photomicrographs using a best fit ellipse determined with imaging software, ImageJ (W. Rasband, U.S. National Institutes of Health, 2005). ImageJ provides several different methods for calculating a best fit ellipse including fit spline, fit ellipse, and convex hull; no noticeable variations in mean vorticity estimates were observed among these methods. The rigid grain and oblique grain shape foliation techniques were used to estimate mean vorticity numbers.

[28] The rigid grain technique involves measuring either the aspect ratio $(R)$ [Wallis et al., 1993] or shape factor $\left(B^{*}\right)$ [Passchier, 1987] of a rigid porphyroblast such as garnet, hornblende, or tourmaline, and the acute angle $(\theta)$ formed between the long axis of the porphyroblast and main foliation, $\mathrm{S}_{\mathrm{a}}$, where

$$
\begin{gathered}
R=M_{\mathrm{x}} / M_{\mathrm{n}} \\
B^{*}=\left(M_{\mathrm{x}}^{2}-M_{\mathrm{n}}^{2}\right) /\left(M_{\mathrm{x}}^{2}+M_{\mathrm{n}}^{2}\right)
\end{gathered}
$$

$M_{\mathrm{x}}$ is the clast long axis and $M_{\mathrm{n}}$ is the clast short axis (Figure 10). Above a critical aspect ratio $\left(R_{\mathrm{c}}\right)$ or critical shape factor $\left(B^{*}\right)$, grains will reach a stable orientation, whereas grains with aspect ratios or critical shape factors below $R_{\mathrm{c}}$ and $B^{*}{ }_{\mathrm{c}}$ will rotate freely. Vorticity is obtained by plotting $R$ versus $\theta$ on a Wallis plot to define $R_{\mathrm{c}}$ and is related to vorticity by [Passchier and Trouw, 2005]

$$
W_{\mathrm{m}}=\left(R_{\mathrm{c}}^{2}-1\right) /\left(R_{\mathrm{c}}^{2}+1\right)
$$




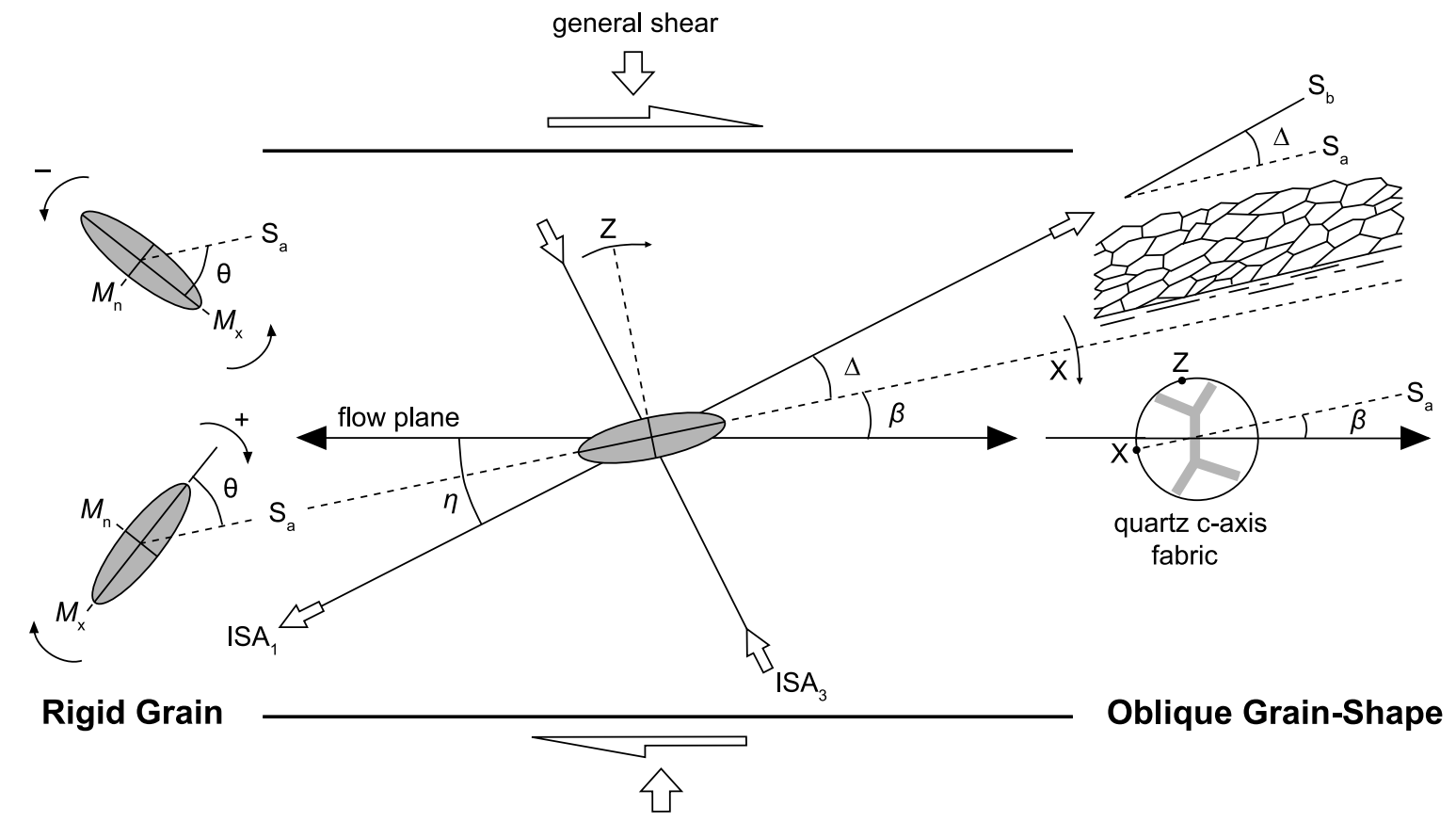

Figure 10. Schematic two-dimensional diagram of forward (positive) and backward (negative) rotated rigid grains used in the rigid grain technique and oblique quartz neoblasts used in the oblique grain shape technique and their orientation relative to instantaneous flow during general shear. The angle $\theta$ defines the angle between the rigid grain long axis $\left(\mathrm{M}_{\mathrm{x}}\right)$ and main foliation $\left(\mathrm{S}_{\mathrm{a}}\right)$. The angle $\delta$ defines the angle between the $\mathrm{Sa}$ and the instantaneous stretching axis $\left(\mathrm{ISA}_{1}\right.$ or $\left.\mathrm{S}_{\mathrm{b}}\right)$. The angle $\beta$ defines the angle between the $\mathrm{S}_{\mathrm{a}}$ and the flow plane. Together, $\beta$ and $\delta$ define the angle $\eta$ between the ISA $\mathrm{A}_{1}$ and the flow plane. $\mathrm{ISA}_{3}$, instantaneous stretching axis; Mn, rigid grain short axis; $\mathrm{X}$ and $\mathrm{Z}$, principal strain axes. Modified from Xypolias [2009] with permission from Elsevier.

or, alternatively, by plotting $B^{*}$ versus $\theta$ on the rigid grain net (Figures 11a and 11b) (see Figure S1 in the auxiliary material for additional plots). ${ }^{1}$ The advantage of the rigid grain net is that vorticity $\left(W_{\mathrm{m}}\right)$ can be read directly from the plot.

[29] To apply the rigid grain method, the following conditions must be met: (1) rigid grains must predate the deformational fabric of interest, (2) rigid grains must be internally undeformed, (3) rigid grains must have rotated independently from the surrounding matrix and other adjacent porphyroblasts, and (4) samples must be characterized by a well-developed LS fabric indicative of plane strain deformation [Jessup et al., 2007]. An inaccurate vorticity determination is likely if the above conditions are not met [Jessup et al., 2007]. In addition, rigid grains in contact with one another during deformation perpendicular to the plane of the thin section will also introduce a bias and uncertainty in the vorticity estimate. Currently, there is no quantitative way to assess these uncertainties associated with mean vorticity estimates obtained from a visual determination of $R_{\mathrm{c}}$ and $B^{*}{ }_{\mathrm{c}}$ (Figure 11$)$. In each sample, all rigid grains that met the above criteria were measured to accurately define $R_{\mathrm{c}}$ and $B^{*}$ and to calculate a mean vorticity number.

\footnotetext{
${ }^{1}$ Auxiliary materials are available in the HTML. doi:10.1029/ $2010 \mathrm{TC} 002746$.
}

[30] The oblique grain shape foliation technique was used on quartzite samples. During progressive deformation, the long axes of quartz neoblasts rotate from an initial parallelism with the instantaneous stretching axis toward the flow plane (Figure 10) [Wallis et al., 1993; Xypolias, 2009]. The oblique grain shape technique involves measuring the maximum angle $\delta$ between the long axis of quartz neoblasts and the main foliation $\mathrm{S}_{\mathrm{a}}$ (Figure 10). Quartz grains that exhibit internal deformation (e.g., associated with GBM) have rotated to a greater or lesser degree compared to quartz neoblasts and should be avoided [Xypolias, 2009]. These angles are then adjusted with respect to the flow plane after measuring the angle $\beta$ between the flow plane and main foliation $\mathrm{S}_{\mathrm{a}}$ from quartz LPOs (Figure 10). The flow plane is defined as the orthogonal to the central girdle of a crossgirdle or single girdle [c] axis LPO (Figure 10) [Wallis, 1992]. Collectively, $\delta+\beta$ define the angle $\eta$ and are related to vorticity by [Xypolias, 2009]

$$
W_{\mathrm{m}}=\sin 2 \eta \text { or } W_{\mathrm{m}}=\sin 2(\delta+\beta)
$$

[31] For this method to be applicable, a well-developed [c] axis fabric and quartz oblique grain shape foliation are needed. To accurately define the ISA, $\delta$ for approximately 100 quartz neoblasts were measured in thin section with respect to the main foliation $S_{a}$ (Figure 10). 


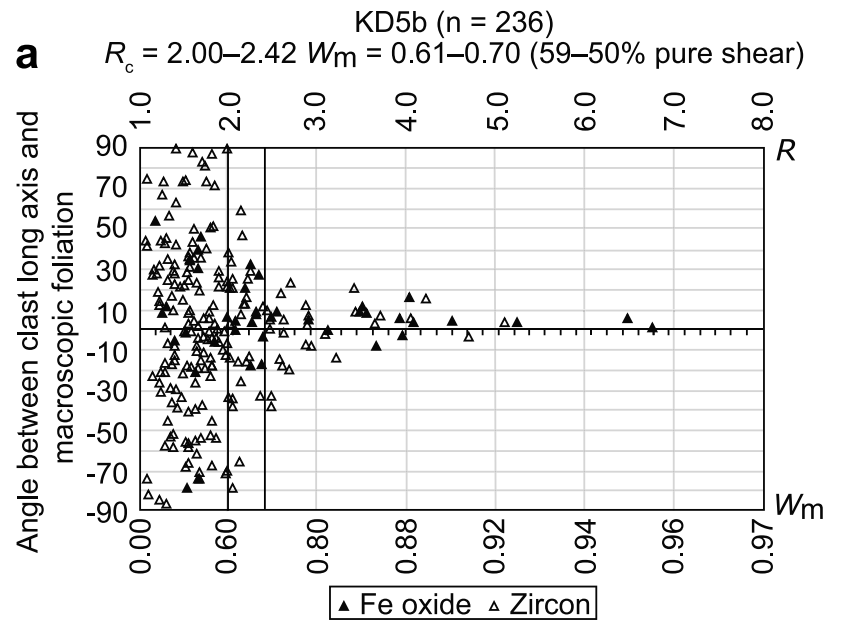

b $\quad W_{m}=0.60-0.72(59-49 \%$ pure shear $)$
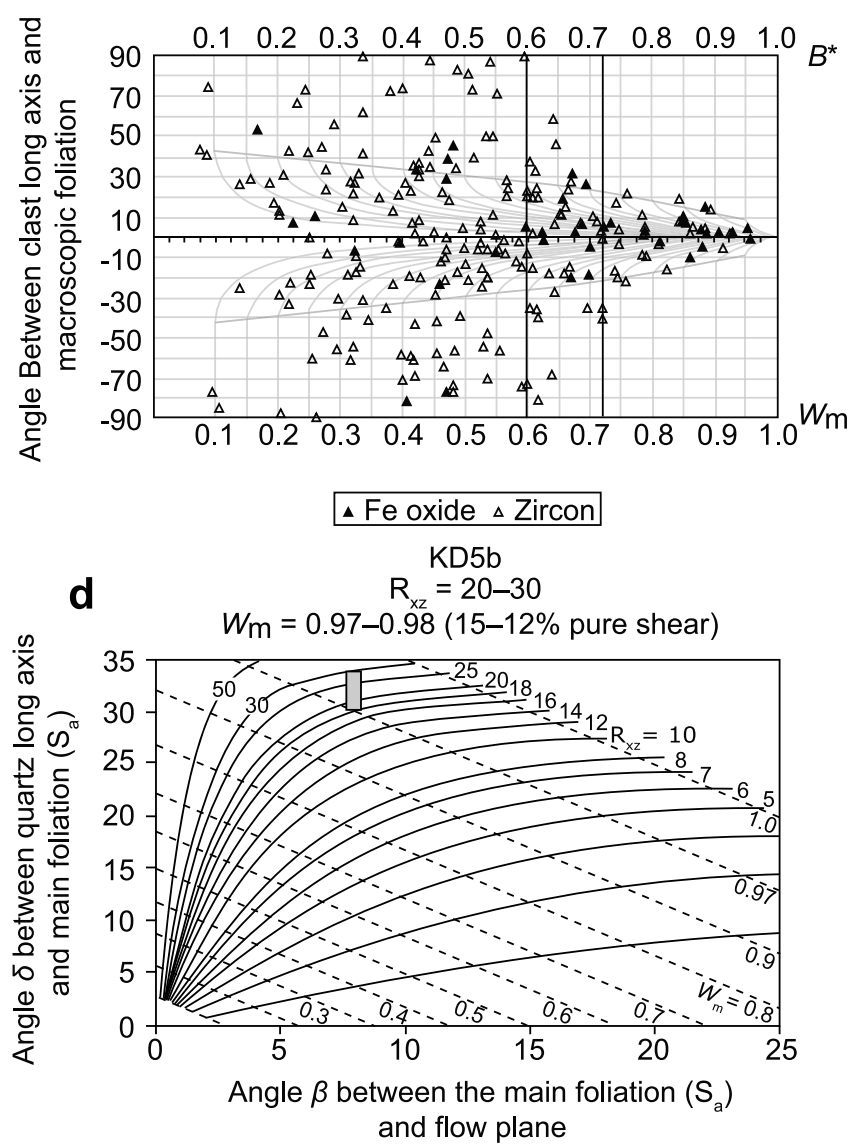

Figure 11. Representative vorticity plots for sample KD5b using multiple techniques. (a) Plot from Wallis et al. [1993] of the angle $\theta$ versus aspect ratio R. The dark vertical lines represent the estimated range for the critical aspect ratio $\mathrm{R}_{\mathrm{c}}$. (b) Rigid grain net [from Jessup et al., 2007] showing a plot of angle $\theta$ versus shape factor $\mathrm{B}^{*}$. The dark vertical lines represent the range for the critical shape factor $\mathrm{B}^{*}{ }_{\mathrm{c}}$ and estimated range of $W_{\mathrm{m}}$. (c) Oblique grain shape plot of frequency versus the angle $\eta$. Vertical dashed lines represent the range in $\eta$ that defines the instantaneous stretching axis (ISA). (d) Plot of the angle $\delta$ versus the angle $\beta$ (see Figure 10) from which strain ratio and vorticity can be calculated [Xypolias, 2009]. Shaded gray box represents the range in $\delta$ versus $\beta$ for sample KD5b. Curved solid lines represent the strain ratio $\left(\mathrm{R}_{\mathrm{xz}}\right)$; diagonal dashed lines represent $W_{\mathrm{m}} ; \mathrm{n}$ is the number of data points. See text for discussion. Additional vorticity plots are shown in Figure S1.

[32] Mean vorticity $\left(W_{\mathrm{m}}\right)$ estimates in schist and orthogneiss samples using the rigid grain technique range from 0.62 to $0.89(57-30 \%$ pure shear) in chloritoid zone rocks, 0.30 $0.85(80-35 \%$ pure shear) in garnet zone rocks, to $0.25-0.76$ (83-45\% pure shear) in staurolite/kyanite zone rocks, and $0.38-0.61$ ( $75-58 \%$ pure shear) in the orthogneiss, the deepest exposed rocks (Table 1) (Figure 12). Quartzite samples using the same technique yielded mean vorticity estimates that are similar: $0.60-0.72(59-49 \%$ pure shear) in chloritoid zone rocks, $0.43-0.67$ (72-53\% pure shear) in garnet zone rocks, and $0.42-0.68$ (72-52\% pure shear) in staurolite/ kyanite zone (Figure 12). Johnson et al. [2009] investigated the reliability of the rigid grain technique for estimating vorticity through the study of naturally deformed samples using feldspar as the measured rigid clast and numerical modeling. Johnson et al.'s [2009] results led them to conclude that mica facilitates porphyroclast/matrix decoupling and that the rigid grain technique applied to mica-rich rocks will likely lead to an incorrect estimate of vorticity that is too low. It seems unlikely that this is an issue in our samples because (1) we did not use feldspar clasts in our measurements, (2) the lowest $W_{\mathrm{m}}$ values recorded in our samples are from those with the lowest percentage of mica, and (3) metamorphic isobars were vertically collapsed to $\sim 20 \%$ of their original thickness by the D2 vertical thinning and horizontal extensional deformation event [Lee et al., 2000].

[33] Only one quartzite sample, KD5b, located in the garnet zone, exhibited a well-defined oblique grain shape foliation and contained enough rigid grains suitable for 
SOUTHERN HALF

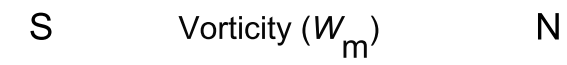

$\begin{array}{llllllll}1 & 0.9 & 0.8 & 0.7 & 0.6 & 0.5 & 0.4 & 0.3\end{array}$

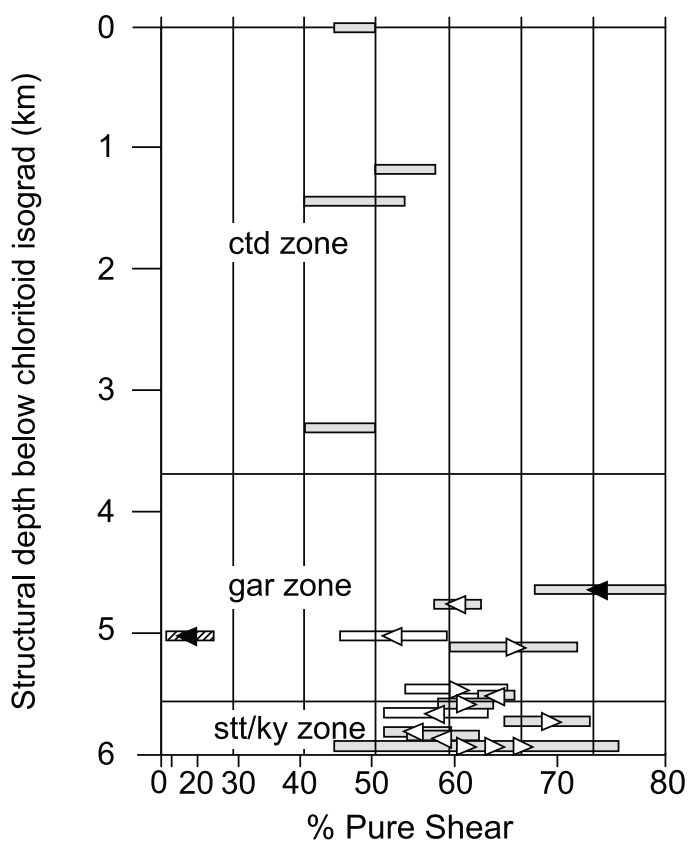

NORTHERN HALF

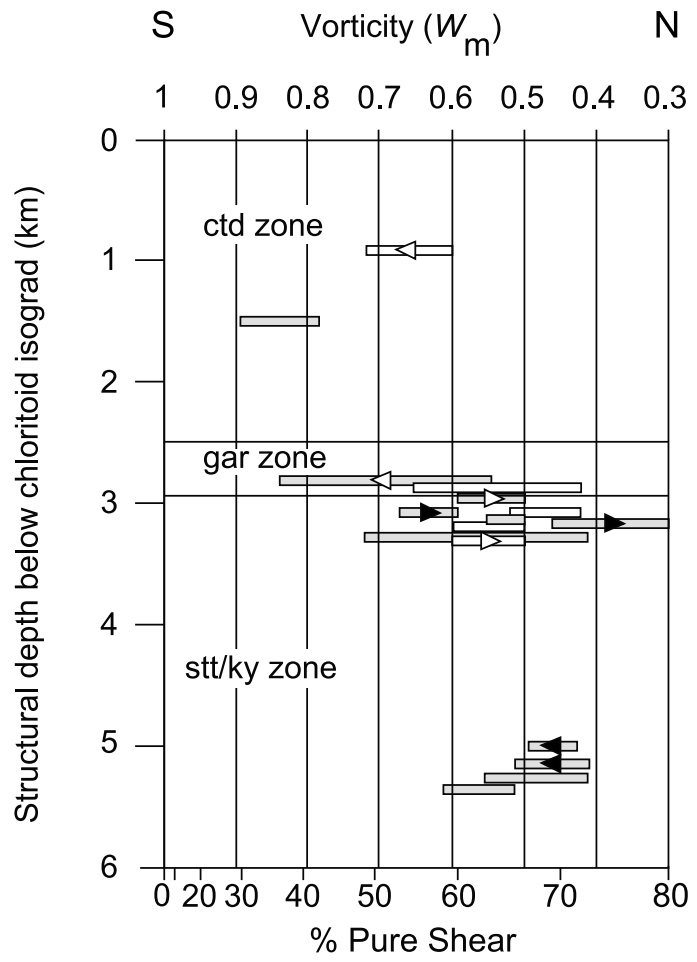

Mean vorticity estimate using the rigid grain technique for orthogneisses, phyllites, and schists

mean vorticy estimate using the oblique grain-shape foliation technique for quartzites

Mean vorticity estimate using the rigid grain technique for quartzites

$\triangleright \quad$ High temperature shear sense indicator

- Low temperature shear sense indicator

Figure 12. Vorticity estimates and shear sense versus structural depth below the chloritoid-in isograd for orthogneiss, phyllite, schist, and quartzite samples. Graphs are shown with respect to cross section B-B' (see Figure 4 for cross section location). Shear sense indicators without a vorticity estimate are not shown.

applying both vorticity techniques. Note that Wallis [1995] suggested that the maximum $\delta$ angle be used to calculate the orientation of the ISA. However, sample KD5b shows a range of quartz elongation orientations, with some exceeding the theoretical maximum of $45^{\circ}$ (Figure 11c). As suggested by Johnson et al. [2009], the range in measured $\delta$ angles could be the result of (1) variations in magnitude of strain recorded in quartz grains as a consequence of dynamic recrystallization and/or (2) rotation relative to the ISA as a consequence of mechanical heterogeneity caused by, for example, porphyroblasts just outside the plane of the section. Because of this, all $\eta$ values greater than $45^{\circ}$ were excluded. To estimate a $\eta$ value for this sample, we calculated a best fit density distribution curve through a frequency versus $\eta$ histogram (Figure 11c). Where the density distribution curve sharply drops and levels off defines the range in angle $\eta$ between the ISA and the flow plane (Figure 11c). The oblique grain shape foliation technique yielded a mean vorticity of $0.93-$ 0.99 (25-9\% pure shear) indicating a large simple shear component, whereas the rigid grain technique yielded a mean vorticity of $0.60-0.75$ (59-46\% pure shear), indicating a larger pure shear component (Figure 11) (Table 1). A mean vorticity of $0.97-0.98$ (15-12\% pure shear) and strain ratio $\left(R_{x z}\right)$ of $\sim 20-30$, in agreement with the bulk strain ratio estimated by Lee et al. [2000], were estimated for this sample using a plot of $\delta$ versus $\beta$ (Figure 11). Advantages of this plot are that it allows a way to check the accuracy of $W_{\mathrm{m}}, \delta, \beta$, and $\eta$ obtained by other techniques; eliminates the assumption of a steady state deformation; and provides an estimate of strain ratio [Xypolias, 2009]. Plots of vorticity and contours of percentage of pure shear (Figures 4, 5, and 12) indicate an increasing component of pure shear with structural depth and with increasing deformation temperature.

[34] There are six salient observations that fall from the kinematic, deformation temperature, and vorticity data. First, relatively high-temperature D2 kinematic indicators show a subequal mix of top-north and top-south shear in garnet zone rocks, and dominantly top-north shear in staurolite/kyanite 
zone rocks and the underlying orthogneiss (Figure 8). Second, relatively low-temperature shear bands are characterized by dominantly top-north shear across the dome (Figure 8). Third, deformation temperatures determined from quartz, feldspar, and calcite microstructures, mineral assemblages from strain shadows of rotated index minerals, and quartz LPOs overlap with peak metamorphic temperature estimates of Lee et al. [2000] (Figure 8). This relation indicates that high-temperature D2 kinematic indicators and fabrics formed at peak metamorphic temperatures. Fourth, quartz and feldspar microstructures indicate that shear bands formed at temperatures that overlap with estimated ${ }^{40} \mathrm{Ar} /{ }^{39} \mathrm{Ar}$ mica closure temperatures $\left(\sim 285\right.$ to $\left.420^{\circ} \mathrm{C}\right)$ [Lee et al., 2000] and are colder than D2 fabric temperatures (Figure 8). Alternatively, increased strain rate may explain the quartz microstructures observed in the shear bands [e.g., Heard and Carter, 1968; Koch et al., 1989]. However, natural strain rates appear to be relatively constant, as a consequence changes in quartz microstructures have typically been attributed to changes in temperature [e.g., Stipp et al., $2002 \mathrm{~b}$, and references therein]. Thus, we conclude that these microstructures recorded formation of relatively lowtemperature kinematic indicators that developed during postpeak cooling after the main, higher temperature, highstrain phase of D2 deformation. Fifth, the percentage of pure shear recorded in these rocks increases with depth from an average of $\sim 48 \%$ pure shear in chloritoid zone rocks to $\sim 62 \%$ pure shear in garnet to staurolite/kyanite zone rocks, to $\sim 68 \%$ in the orthogneiss. Sixth, both high- and low-temperature D2 ductile fabrics disappear within the orthogneiss $\sim 1000 \mathrm{~m}$ below the contact with the overlying metasediments. Thus, mylonitic D2 deformational fabrics define an $2200-2900 \mathrm{~m}$ thick zone extending from the garnet-in isograd [Lee et al., 2000, 2002] to $1000 \mathrm{~m}$ below the contact between the orthogneiss and overlying metasediments (Figure 5).

\section{Discussion}

\subsection{Deformation Patterns in the Midcrust}

[35] The relatively high temperature D2 kinematic fabrics in midcrustal rocks exposed in Kangmar Dome yield a dominantly top-north D2 kinematic fabric and a pure shear deformation component that increases with depth. This kinematic and vorticity pattern suggests that deformation across the dome was characterized by general shear (vertical thinning and $\mathrm{N}-\mathrm{S}$ horizontal extension) with a component of top-north sense of shear. The pattern of D2 kinematics across Kangmar documented in this study differs from the bulk pure shear kinematic interpretation by Lee et al. [2000] and contrasts with the solely top-north shear discussed by Chen et al. [1990]. It is consistent with the kinematic pattern of deformation predicted by a combination of the top half of Poiseuille flow (Figure 2b) and a shear zone parallel stretch, the implied pattern of midcrustal deformation predicted by the channel flow hypothesis [e.g., Beaumont et al., 2004; Grujic et al., 2002].

[36] High-strain mylonitic fabrics are exposed within metasedimentary and orthogneissic rocks in the core of Kangmar dome from the garnet-in isograd, $\sim 1200-1900 \mathrm{~m}$ above the contact between the two to $\sim 20 \mathrm{~m}$ below. The strength of these fabrics within the orthogneiss progressively decreases with increasing structural depth beneath the contact for about $\sim 1000 \mathrm{~m}$, below which the orthogneiss appears undeformed. Above the garnet-in isograd, the strength of D2 fabrics progressively decreases up section for $\sim 650-1450 \mathrm{~m}$, above which the metasedimentary rocks do not possess a D2 fabric. Thus, the mylonitic fabrics define a high-strain D2 deformation zone 1200-1900 m thick that straddles the contact between the orthogneiss core and its metasedimentary mantle. This high-strain zone is located within a much wider, a 2200-2900 m thick D2 deformation zone that extends from the approximate level of the garnet-in isograd, where the S1 foliation has been completely transposed into parallelism with the S2 mylonitic foliation [Lee et al., 2000], to $\sim 1000 \mathrm{~m}$ below the contact between the orthogneiss and overlying metasediments (Figure 5).

[37] The contact between the orthogneiss core and metasedimentary mantle in Kangmar has been interpreted as an intrusive contact [Hayden, 1912; Zhou et al., 1981], a metamorphic core complex-type detachment fault [Chen et al., 1990], and an unconformity [Burg et al., 1984; Zhang et al., 1986; Lee et al., 2000]. Combining our observations of a high-strain structural fabric above and below the contact with Lee et al.'s [2000] description of the contact as varying from a knife-sharp concordant contact to a locally discordant contact with up to $\sim 1 \mathrm{~m}$ of breccia and fault gouge, suggests that the orthogneiss-metasedimentary contact is a fault. Because of the dominance of top-north shear in the ductilely deformed metapelites above and the orthogneiss below, we concur with the suggestion of that Chen et al. [1990] and Hauck et al. [1998] that this fault is the northern continuation of the STDS. A consequence of this interpretation is that Kangmar Dome records a progressive deformation history characterized by an initial period of general shear with a top-north simple shear component at relatively high temperatures and midcrustal depths ( 20-30 km [Lee et al., 2000]), followed by general shear with a dominantly top-north simple shear component at somewhat lower temperatures and shallower depths, ending with both sets of ductile fabrics subsequently cut by the top-north normal slip STDS as these midcrustal rocks were exhumed to shallower crustal levels. Slip along this brittle structure resulted in the juxtaposition of high-grade metapelites upon the orthogneiss. In this interpretation, the absence of a break in mica ${ }^{40} \mathrm{Ar} /{ }^{39} \mathrm{Ar}$ ages across the fault contact [Lee et al., 2000] can be explained by (1) reheating and cooling of the rocks after faulting, (2) slip above mica closure temperature [e.g., Chen et al., 1990], or (3) smallmagnitude fault slip. The first interpretation is likely implausible because no source of heat, such as a pluton or dike swarm, has been observed in Kangmar [Burg et al., 1984; Chen et al., 1990; Lee et al., 2000, 2002] and footwall rocks below the fault contact yield older mica ${ }^{40} \mathrm{Ar} /{ }^{39} \mathrm{Ar}$ cooling ages [Lee et al., 2000]. The second interpretation also is unlikely because the microstructures documented in this paper show that faulting occurred after the development of the moderate temperature D2 shear bands. The third interpretation is most plausible because the 
same lithologic units and metamorphic grade are juxtaposed across the $\sim 10 \mathrm{~km}$ long exposure of the fault.

[38] The North Himalayan gneiss domes, Malashan, Mabja, Kampa, and Kangmar, preserve a midcrustal D2 extensional deformation event that is late Eocene/early Oligocene to middle Miocene in age [Chen et al., 1990; Aoya et al., 2005, 2006; Kawakami et al., 2007; Quigley et al., 2006, 2008; Lee et al., 2000, 2004, 2006; Lee and Whitehouse, 2007; Langille et al., 2010]. However, the kinematics and structural depth associated with this high-strain D2 extensional deformation varies from dome to dome. In Malashan Dome (Figure 1), Aoya et al. [2005, 2006] documented dominantly top-north shear, and associated this style of deformation with extension triggered by the emplacement of granites within the middle to upper structural levels of the Tethys Himalaya (depths of $\sim 10 \mathrm{~km}$ [Kawakami et al., 2007]) above the STDS. The D2 sense of shear in Kampa Dome (Figure 1), recorded in metasediments that reached kyanite zone metamorphic grade (depths of $>16 \mathrm{~km}$ ), was also dominantly top-north, but was attributed to crustal extension related to slip along the STDS [Quigley et al., 2006, 2008]. In contrast, D2 kinematics within midcrustal rocks exposed in the core of Mabja Dome (depths of $>20-25 \mathrm{~km}$ [Lee et al., 2004]) (Figure 1) change from mixed top-north and topsouth shear at moderate structural levels, through dominantly top-south shear within deep structural levels, to solely topsouth shear in the deepest rocks studied [Langille et al., 2010] (Figure 3). Langille et al. [2010] attributed this pattern of ductile deformation to a hybrid flow regime characterized by spatial variations in viscosity and/or bulk pure shear at moderate structural levels and underthrusting beneath the MHT and/or extrusion of middle crust at the deepest structural levels. These relations imply that midcrustal ductile deformation in southern Tibet varied spatially and rheologically, and indicate along-strike changes in the depth of midcrustal ductile extension. This in turn suggests that either (1) the assumption that the Tethys Himalaya was the rigid upper crust above a flowing ductile middle crust is not valid [e.g., Searle et al., 2003; Beaumont et al., 2004; Kawakami et al., 2007] or (2) the multiple faults that define the STDS in the high Himalaya [e.g., Searle et al., 2003; Godin et al., 2006; Kellett et al., 2009] may not merge northward and downward into a single fault.

[39] In Malashan, Mabja, and Kampa domes, the top of the D2 deformation zone has been documented, however, the base of the deformation zone has not. The D2 fabrics in Malashan span a structural thickness of at least $\sim 2500$ $3000 \mathrm{~m}$ (from Middle Jurassic metapelites to the underlying early Miocene Malashan granite [Aoya et al., 2005, 2006; S. Wallis, personal communication, 2010]). In Mabja Dome, D2 ductile fabrics extend continuously from the base of Triassic metapelites to the deepest exposures mapped, indicating a D2 deformation zone more than $\sim 7500 \mathrm{~m}$ thick [Lee et al., 2004]. At the highest structural levels in Kampa Dome, D2 ductile fabrics are exposed from the middle of Triassic metapelites to the deepest exposures yielding a minimum estimate for thickness of the D2 deformation zone of $\sim 2050 \mathrm{~m}$ [Quigley et al., 2006, 2008]. The D2 deformation zones documented in these gneiss domes represent the ductile equivalent of the broad brittle deformation zone of the STDS and the ductile deformation in its footwall. For example, in the Mount Everest region and in Bhutan, mapping has documented two normal-slip detachment faults separated by a kilometer or two and footwall ductile deformation distributed across a structural thickness of 3-4 km [e.g., Grujic et al., 2002; Searle et al., 2003; Law et al., 2004; Jessup et al., 2006; Kellett et al., 2009].

[40] Vorticity estimates in Kangmar associated with D2 deformation decrease with structural depth, indicating an increase in pure shear component from $\sim 48 \%$ in chloritoid zone rocks, through $\sim 62 \%$ in garnet zone to staurolite/kyanite zone rocks, to $\sim 68 \%$ in the orthogneiss, the deepest exposed rocks (Figures $4 \mathrm{~d}, 5 \mathrm{~d}$, and 12) (Table 1). An increase in pure shear with depth indicates significant vertical thinning and horizontal extension, consistent with the documented welldeveloped subhorizontal D2 foliation and stretching lineation, the rotation of D1 linear structures into parallelism with the D2 stretching lineation, and the collapse of metamorphic isobars to $\sim 20 \%$ of their original thickness [Lee et al., 2000]. We ascribe the increase in pure shear with depth, at least in part, to an increased lithostatic load, the same mechanism proposed for the increase in pure shear with structural depth documented in midcrustal rocks exposed on Mount Everest [Law et al., 2004; Jessup et al., 2006] and in Mabja Dome [Langille et al., 2010].

[41] In Kangmar, deformation temperatures associated with relatively high-temperature D2 kinematic fabrics increase with structural depth from $\sim 300-500^{\circ} \mathrm{C}$ in chloritoid zone rocks to $>630^{\circ} \mathrm{C}$ in staurolite/kyanite zone and deeper rocks. These deformation temperature estimates overlap with quantitative peak metamorphic temperature estimates of Lee et al. [2000] (Figure 8), indicating that D2 kinematics and vorticity were recorded during peak metamorphism prior to dome formation and exhumation. The onset of the D2 high-temperature event is not constrained in this study, but is late Eocene/early Oligocene in Mabja Dome [Lee and Whitehouse, 2007], which records deformational and metamorphic histories similar to Kangmar [cf. Lee et al., 2000; Lee et al., 2004, 2006]. Based on ${ }^{40} \mathrm{Ar} /{ }^{39} \mathrm{Ar}$ mica ages, D2 deformation ceased by $\sim 15 \mathrm{Ma}$, indicating that temperatures had dropped below $\sim 280-420^{\circ} \mathrm{C}$ at this time [Lee et al., 2000]. A middle Miocene cessation age is similar to the documented end of the same high-temperature D2 extensional deformational event in Malashan [Aoya et al., 2005], Mabja [Lee et al., 2006; Lee and Whitehouse, 2007], and Kampa [Quigley et al., 2006] domes.

[42] Relatively low-temperature shear bands in garnet zone and deeper rocks are characterized by dominantly topnorth shear across Kangmar. Deformation temperatures for these kinematic indicators overlap with estimated mica ${ }^{40} \mathrm{Ar}{ }^{39} \mathrm{Ar}$ closure temperature estimates $\left(\sim 280-420^{\circ} \mathrm{C}\right)[$ Lee et al., 2000] (Figure 8), indicating that these shear bands formed at $\sim 15 \mathrm{Ma}$. Contours of middle Miocene mica ${ }^{40} \mathrm{Ar}{ }^{39} \mathrm{Ar}$ ages are domed [Lee et al., 2000], implying that this low-temperature deformation event occurred during cooling and exhumation after peak metamorphism and after the high-strain D2 deformation, but likely shortly before brittle slip along the STDS and doming. Lee et al. [2000] suggested that subsequent doming was the consequence of capture of midcrustal rocks in the hanging wall of the north 
dipping GKT and southward slip up and over a midcrustal ramp.

[43] Based on the data presented here, data from structure and metamorphic petrology from Kangmar [Lee et al., 2000], and geochronology from Kangmar [Lee et al., 2000], Kampa [Quigley et al., 2006, 2008], Mabja [Lee et al., 2006; Lee and Whitehouse, 2007], and Malashan [Aoya et al., 2005], we propose the following evolution for Kangmar Dome that is a slightly modified version of that postulated by Lee et al. [2000]. During the late Eocene/early Oligocene to early/middle Miocene, brittle normal slip along the STDS merged at depth to the north into a zone of ductile shear, which, in turn, merged into a $\sim 2-3 \mathrm{~km}$ wide root zone of general shear in the midcrust (Figure 13a). Pure shear vertical thinning and $\mathrm{N}-\mathrm{S}$ horizontal stretching combined with top-north simple shear in the midcrust of southern Tibet, accommodated at shallow crustal levels to the south by normal slip along the STDS, resulted in partial exhumation of these midcrustal rocks by the early to middle Miocene (Figure 13b). During the middle Miocene, periods of either high strain rates along the STDS and/or continued exhumation and cooling of the midcrustal rocks resulted in propagation of the brittle STDS into the zone of general shear (Figure 13b). Thus, the brittle STDS cut down structural section northward from the high Himalaya where, in the Everest region for example, it juxtaposed unmetamorphosed Indian margin sedimentary rocks in the hanging wall upon the GHS in the footwall, to Kangmar where it juxtaposed metamorphosed Tethyan sediments in the hanging wall upon an orthogneiss in the footwall. Slip along the STDS ceased by the middle Miocene [Murphy and Harrison, 1999; Godin et al., 2006, and references therein; Kellett et al., 2009]. As postulated by Lee et al. [2000], soon thereafter middle to late Miocene south directed thrust faulting along the GKT and erosion resulted in doming and final exhumation of the midcrustal rocks to Earth's surface (Figures 13c and 13d).

\subsection{Tectonic Implications}

[44] Much of the structural, metamorphic, and intrusive histories recorded in midcrustal rocks exposed in the cores of the North Himalayan gneiss domes are strikingly similar to the histories recorded in the midcrustal GHS exposed in the high Himalaya [cf. Murphy and Harrison, 1999; Vance and Harris, 1999; Walker et al., 1999; Simpson et al., 2000; Searle et al., 2003; Law et al., 2004; Jessup et al., 2006; Aoya et al., 2006; Lee et al., 2000, 2006; Lee and Whitehouse, 2007; Kawakami et al., 2007; Quigley et al., 2008; Cottle et al., 2009; Langille et al., 2010]. These similarities suggest that since the late Eocene/early Oligocene to middle Miocene, vertically thinning and horizontally stretching midcrustal rocks were contiguous for at least $\sim 100-150 \mathrm{~km}$ from southern Tibet southward to the high Himalaya [e.g., Grujic et al., 1996, 2002; Beaumont et al., 2001, 2004; Searle et al., 2003; Lee et al., 2000, 2006; Lee and Whitehouse, 2007; Cottle et al., 2009]. Geochronologic studies show that ductile shear and brittle slip along the MCT and STDS were broadly synchronous [e.g., Hodges et al., 1992, 1996; Murphy and Harrison, 1999;
Grujic et al., 1996; Searle et al., 2003], initiating as early as late Eocene/early Oligocene [Lee and Whitehouse, 2007; Cottle et al., 2009] and ending during the middle Miocene [e.g., Hodges et al., 1996; Murphy and Harrison, 1999; Lee and Whitehouse, 2007; Cottle et al., 2009; Kellett et al., 2009], an 20 Myr history. Thus, midcrustal rocks of the GHS and the North Himalayan gneiss domes experienced vertical thinning and horizontal extension between the overlying normal sense STDS and underlying thrust sense MCT, or their precursors, for $20 \mathrm{Myr}$ from the late Eocene/ early Oligocene to the middle Miocene.

[45] The kinematic and vorticity patterns reported here for Kangmar Dome, combined with kinematic and vorticity patterns from Mabja Dome [Langille et al., 2010] and the GHS [Grasemann et al., 1999; Law et al., 2004; Jessup et al., 2006; Carosi et al., 2006, 2007; Larson and Godin, 2009], define general shear deformation characterized by top-north simple shear near the STDS, increasing pure shear with depth, and top-south general shear near the MCT. Although the MCT has not been mapped within Mabja Dome [Lee et al., 2004] and is not exposed in Kangmar [Lee et al., 2000, 2002], the top-south shear documented at the deepest structural levels within Mabja [Langille et al., 2010] suggests that it is present just below the deepest mapped exposures, and we speculate that it must be present beneath Kangmar. This ductile deformation pattern is broadly consistent with geometric and kinematic models proposed to explain the southward extrusion of middle crustal rocks bounded above and below by two opposing sense shear zones, the STDS and MCT [e.g., Grasemann et al., 1999; Vannay and Grasemann, 2001; Williams et al., 2006] (Figure 14a). In these models, flow within an extruding wedge or slab is characterized by simple shear deformation along the boundaries of the wedge grading through general shear toward the center of the wedge that extruded by pure shear.

[46] Seismic reflection data have been interpreted to show that the STDS and MCT converge to the north [Nelson et al., 1996; Hauck et al., 1998] (but see Makovsky et al. [1996]). If this same geometry prevailed during the late Eocene to middle Miocene, the combination of the wedge shape and strain compatibility requires southward extrusion and general shear deformation of midcrustal rocks bounded by the two opposing sense shear zones [e.g., Ramsay and Huber, 1987; Grasemann et al., 1999; Vannay and Grasemann, 2001]. Strain compatibility also requires southward extrusion of a midcrustal slab if the STDS and MCT did not converge to the north [e.g., Makovsky et al., 1996] and Asia acted as a lithostatic backstop. The heterogeneous general shear deformation (characterized by an increasing pure shear (vertical thinning perpendicular and horizontal stretching parallel to the shear zone boundaries) component with structural depth) documented across the midcrust in the GHS [Grasemann et al., 1999; Law et al., 2004; Jessup et al., 2006; Carosi et al., 2006, 2007; Larson and Godin, 2009] and the midcrust in southern Tibet [this study; Langille et al., 2010] is consistent with both the wedge and slab geometric kinematic configurations. Moreover, heterogeneous pure shear perpendicular to the midcrustal boundaries and southward extrusion would have 

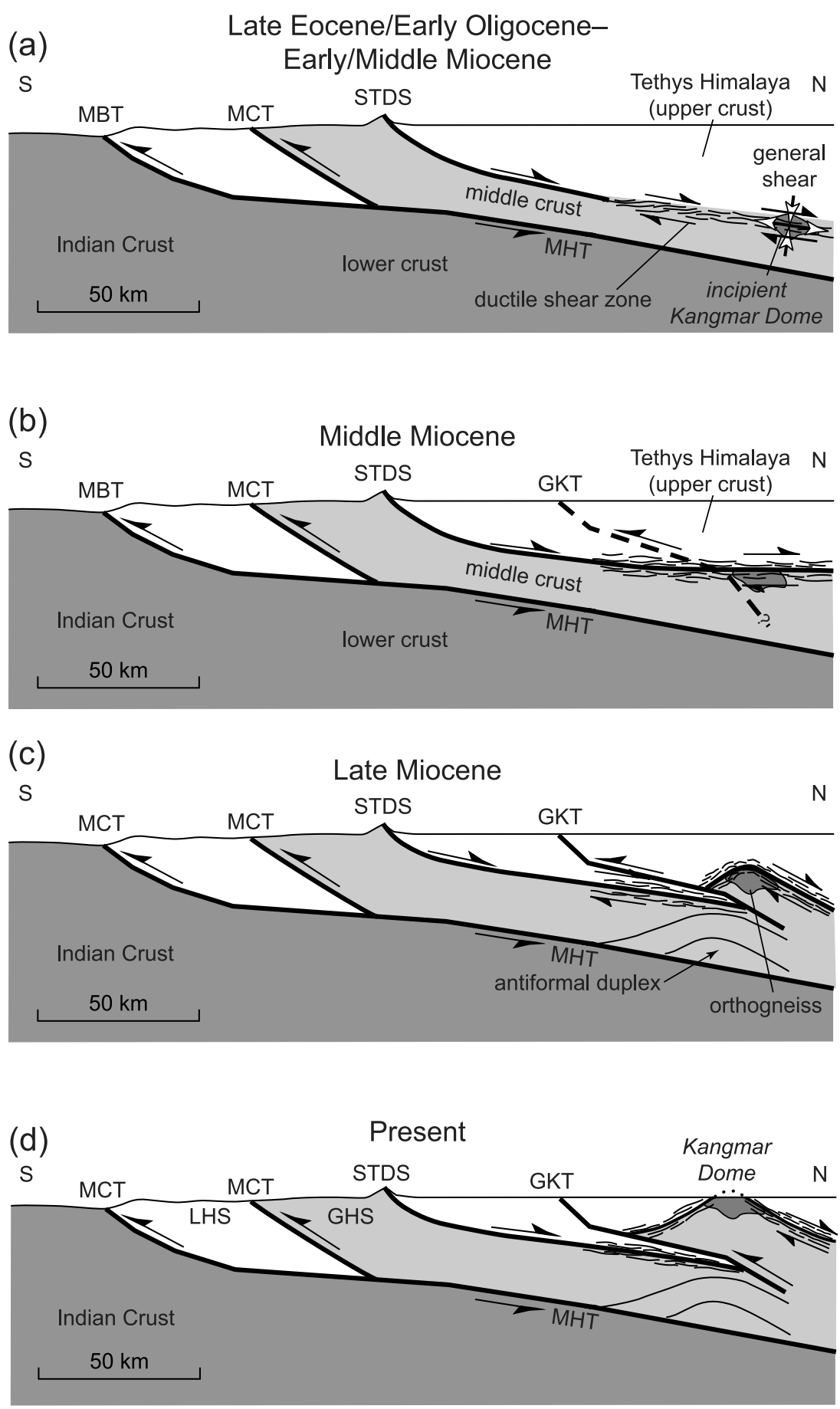

Figure 13. Schematic, interpretative, evolutionary N-S cross sections at the longitude of Kangmar Dome. (a) Configuration of major structures and D2 extensional fabrics in the middle crust of southern Tibet from onset of D2 deformation to near its cessation. (b) Episodes of either high strain rates along the STDS and/or exhumation and cooling of the middle crustal rocks resulted in propagation of STDS into the zone of ductile general shear. (c and d) As postulated by Lee et al. [2000], late Miocene south vergent thrust faulting along the GKT and erosion resulted in doming and final exhumation of the midcrustal rocks to the Earth's surface. GHS, Greater Himalayan sequence; GKT, Gyirong-Kangmar thrust fault system; LHS, Lesser Himalayan sequence; MBT, Main Boundary thrust; MCT, Main Central thrust; MHT, Main Himalayan thrust; STDS, southern Tibetan detachment system. After Lee et al. [2000]. 
a

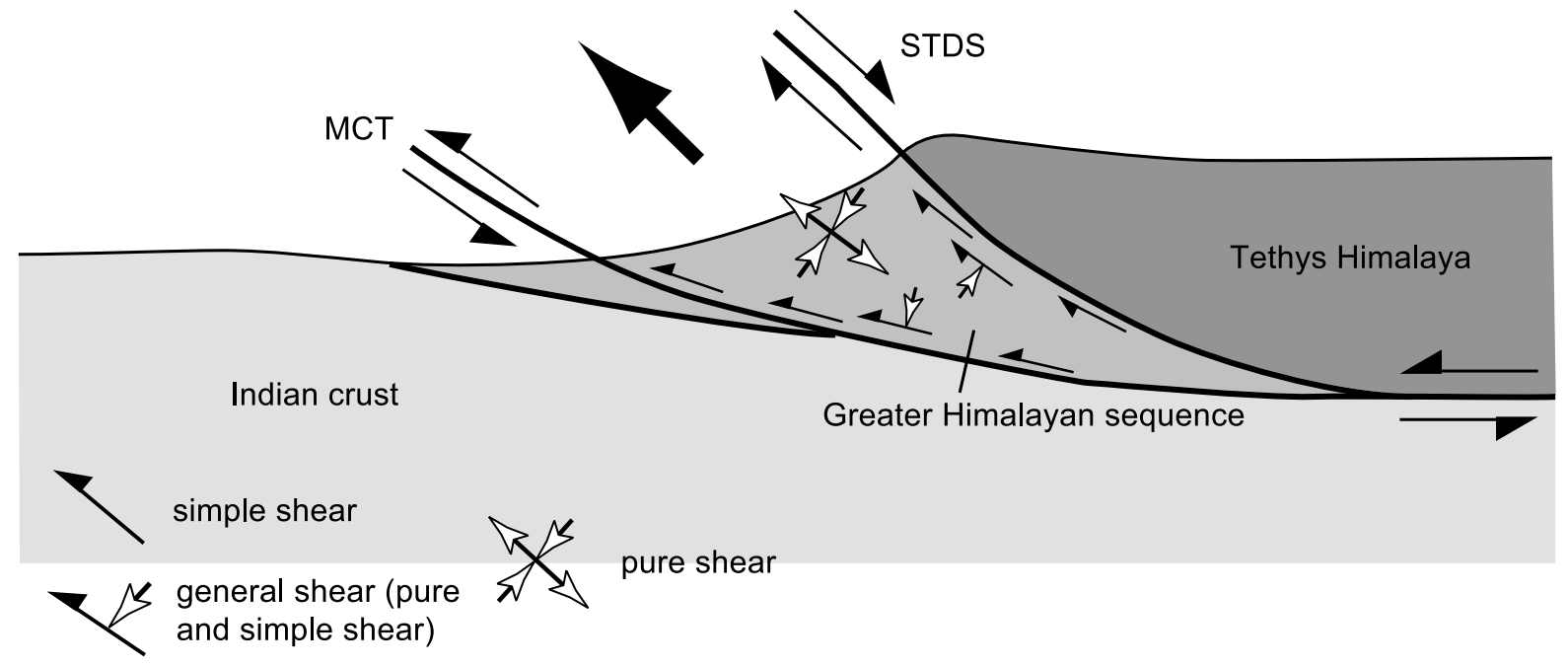

b
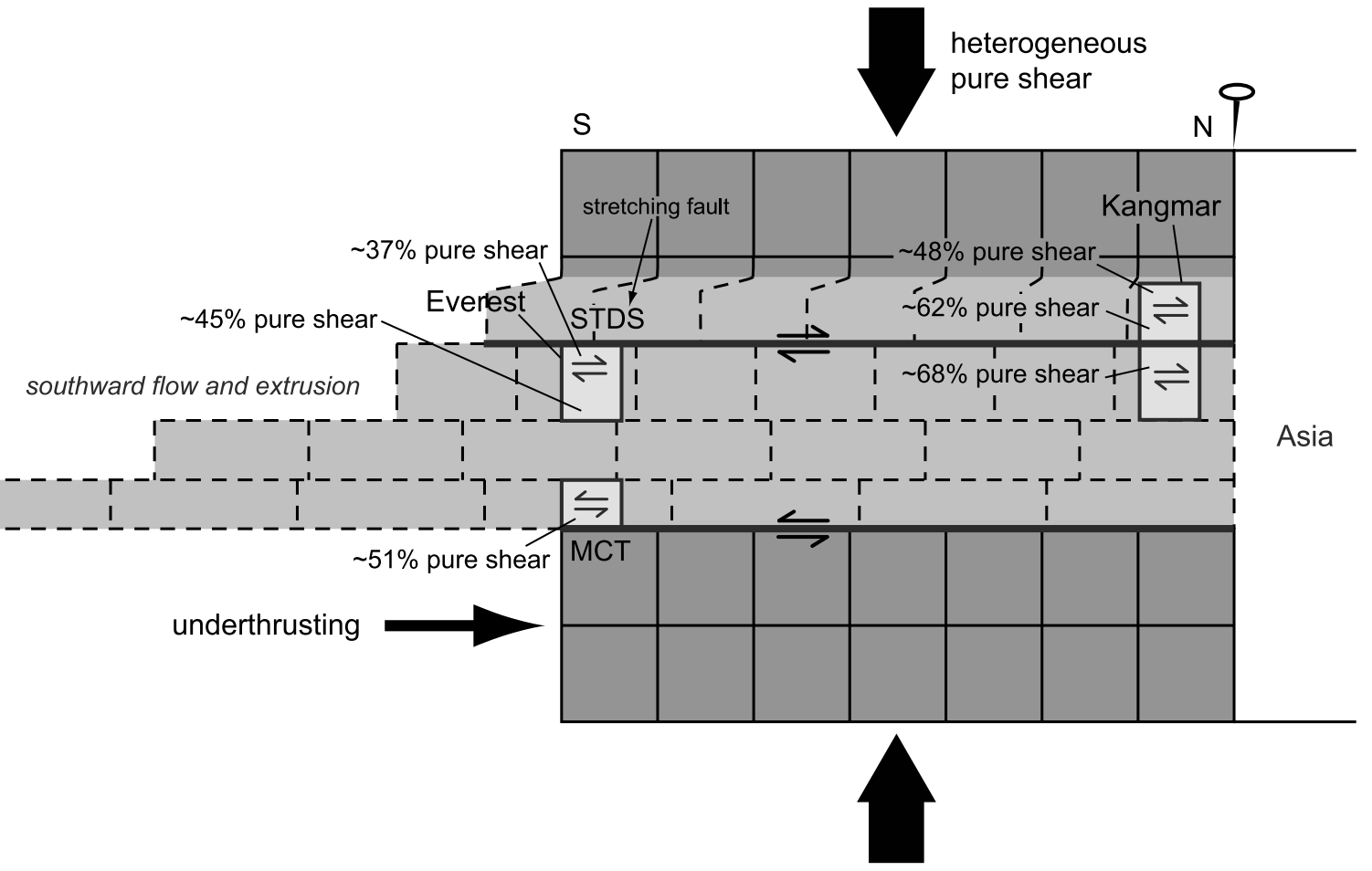

Figure 14. (a) Schematic cross section showing extrusion of a ductile wedge-shaped block deforming by opposing simple shear at the boundaries, general shear toward the middle, and pure shear within the middle. After Grasemann et al. [1999]. (b) Schematic cross section showing how heterogeneous pure shear across midcrustal rocks (light gray) in southern Tibet and the high Himalaya drives southward ductile flow and extrusion and development of the opposing shear sense along the STDS and MCT. Dashed rectangles in the middle crust are schematic strain markers compared to the unstrained solid squares above and below the middle crust. Arrow pairs indicate sense of shear. Pale gray boxes show relative locations of the midcrustal GHS exposed in the Everest massif and midcrustal rocks exposed in the core of Kangmar Dome. Percent pure shear values are from Law et al. [2004], Jessup et al. [2006], and this work. Dark gray boxes in the hanging wall of the STDS and the footwall of the MCT are rigid. See text for discussion. Modified from Langille et al. [2010] with permission from Elsevier. 
caused a southward increase in the magnitude of stretching [e.g., Pfiffner and Ramsay, 1982; Ramsay and Huber, 1987]. The manifestation of this would be an increase in displacement from north to south along the STDS and the underlying MHT (Figure 14b). The lack of evidence for significant slip along the STDS in Kangmar compared to the 90-216 km of southward displacement estimated for the GHS in the footwall of the STDS at the Everest massif [Searle et al., 2003] supports this postulate.

[47] The midcrustal channel flow hypothesis predicts that deformation patterns recorded within a midcrustal channel will be the result of mixed Poiseuille flow and Couette flow [Beaumont et al., 2001, 2004; Grujic et al., 2002; Grujic, 2006]. Poiseuille flow will be favored by a reduction in viscosity within the midcrust, a decrease in the rate of convergence between India and Asia, an increase in channel thickness, and an increase in the subhorizontal pressure gradient along the channel; the opposites favor Couette flow [Turcotte and Schubert, 2002]. Poiseuille-dominated flow in the Himalaya is suggested by (1) low-viscosity midcrust, which is implied by syntectonic migmatites and leucogranite melts in the GHS and within Malashan and Mabja domes [e.g., Murphy and Harrison, 1999; Searle et al., 2003; Aoya et al., 2005, 2006; Lee et al., 2004; Lee and Whitehouse, 2007], (2) the reduction in convergence rate between India and Asia since collision, including dramatic decreases at 45-40 Ma [Molnar and Stock, 2009] or, based on force balance calculations, $\sim 50-35 \mathrm{Ma}$ [Copley et al., 2010], and (3) a large horizontal gravitational potential energy gradient between the Tibetan Plateau and its margins because of the potential of significant relief or crustal thickness during the early stages of D2 deformation (late Eocene to early Oligocene) [e.g., Rowley and Currie, 2006]. However, Couettedominated flow is suggested by (1) a decrease in channel thickness implied by the documented $\sim 30-70 \%$ pure shear deformation component perpendicular to shear boundaries in midcrustal rocks exposed in the core of Kangmar (this study) and Mabja [Langille et al., 2010] domes, and the GHS exposed on Mount Everest [Law et al., 2004; Jessup et al., 2006], in Nepal [Carosi et al., 2007; Larson and Godin, 2009], in Bhutan [Carosi et al., 2006], and northwestern India [Grasemann et al., 1999] and (2) a higher viscosity in migmatite- and leucogranite-absent midcrust, as appears to be the case in Kangmar and Kampa domes [Chen et al., 1990; Lee et al., 2000, 2002; Quigley et al., 2006, 2008] and the lower part of the GHS [e.g., Grasemann et al., 1999; Larson and Godin, 2009]. The potential combination of Poiseuille and Couette flow suggests that these rocks record a hybrid flow regime.

[48] In addition to evidence implying combined Poiseuille and Couette flow, kinematic and vorticity data suggest temporal and spatial variations in the distribution of the deformation field. For example, Grasemann et al. [1999] proposed that the flow regime at the base of the GHS was temporally partitioned following a decelerating strain path, and Jessup et al. [2006] concluded that flow recorded in the GHS on the Everest massif was spatially and temporally partitioned such that structurally lower, higher-temperature samples recorded early stages of ductile general shear and structurally higher, lower temperature samples recorded subsimple shear at the upper boundary of the channel during later stages of exhumation. Variations in rheology may also play a role. Langille et al. [2010] postulated that midcrustal rocks in Mabja Dome recorded a hybrid flow regime, characterized by an overlap of Poiseuille flow, concentrated at higher structural levels, and Couette flow, concentrated at deeper structural levels, in part a consequence of spatial variations in viscosity. If these interpretations are correct, these comparisons suggest that patterns of midcrustal flow in southern Tibet and the high Himalaya varied spatially, temporally, and with rheology.

\section{Conclusions}

[49] New detailed microstructural kinematic analyses, deformation temperature estimates, and vorticity investigations within midcrustal rocks exposed in the core of Kangmar Dome identify a 2200-2900 m thick high-strain zone characterized by vertical thinning and $\mathrm{N}-\mathrm{S}$ horizontal extension. Kinematic analyses show a downward transition from a subequal mix of top-north and top-south shear in garnet zone rocks to dominantly top-north shear in staurolite/kyanite zone and deeper rocks. The schists and quartzites in chloritoid, garnet, and staurolite/kyanite zone rocks record general shear deformation characterized by a pure shear component (vertical thinning and $\mathrm{N}-\mathrm{S}$ horizontal extension) and a top-north simple shear component. Vorticity values progressively decrease down structure from $W_{\mathrm{m}}=$ 0.60-0.89 (59-30\% pure shear) in chloritoid zone rocks, $W_{\mathrm{m}}=0.30-0.85(80-35 \%$ pure shear) in garnet zone rocks, to $W_{\mathrm{m}}=0.25-0.76$ (83-45\% pure shear) in staurolite/kyanite zone rocks. At the deepest exposed structural levels, the orthogneiss also records general shear deformation $\left(W_{\mathrm{m}}=\right.$ $0.38-0.61 ; 75-58 \%$ pure shear, the highest recorded in Kangmar) with a moderate component of top-north simple shear. Deformation temperature estimates for high-temperature kinematic indicators increase with structural depth from 350 to $450^{\circ} \mathrm{C}$ in chloritoid zone rocks to $\sim 500-630^{\circ} \mathrm{C}$ is staurolite/kyanite zone rocks and $\geq 600^{\circ} \mathrm{C}$ in the orthogneiss. These temperature estimates overlap with metamorphic petrology temperature estimates, indicating that the vorticity recorded in these rocks occurred in the midcrust during and after D2 deformation, likely after the late Eocene/ early Oligocene and certainly before the middle Miocene. The $\sim 2-3 \mathrm{~km}$ thick ductile deformation zone was subsequently cut by the down-to-the-north brittle normal-slip STDS that juxtaposed metasedimentary rocks upon the orthogneiss. Strain compatibility and an increasing component of pure shear with depth require that these midcrustal rocks were extruded southward. We interpret these relations as indicating ductile deformation within a wedge- or slabshaped midcrust, broadly consistent with patterns predicted by kinematic and thermal-mechanical models. Comparing studies of ductile deformation in midcrustal rocks in southern Tibet and the GHS reveals similarities and differences, suggesting that patterns of midcrustal flow during the Himalayan orogeny varied spatially, temporally, and with rheology. 
[50] Acknowledgments. We thank J. Langille for discussions about her studies on midcrustal rocks exposed in the core of Mabja Dome, S. Wallis for discussions on the interpretation of LPOs, and S. Wallis and S. Johnson on how best to calculate vorticity using the oblique grain shape foliation technique. Reviewers M. Jessup, R. Thiede, and the Associated
Editor provided thoughtful reviews that improved the clarity of this manuscript. This research was supported by NSF grants EAR-0838146 awarded to J. Lee and EAR-0838264 awarded to B. Hacker.

\section{References}

Aoya, M., S. R. Wallis, K. Terada, J. Lee, T. Kawakami, Y. Wang, and M. Heizler (2005), North-south extension in the Tibetan crust triggered by granite emplacement, Geology, 33, 853-856, doi:10.1130/ G21806.1.

Aoya, M., S. R. Wallis, T. Kawakami, J. Lee, Y. Wang, and H. Maeda (2006), The Malashan gneiss dome in south Tibet: Comparative study with the Kangmar Dome with special reference to kinematics of deformation and origin of associated granites, in Channel Flow, Ductile Extrusion and Exhumation in Continental Collision Zones, edited by R. D. Law et al., Geol. Soc. Spec. Publ., 268, 471-495.

Armijo, R., P. Tapponnier, J. L. Mercier, and H. TongLin (1986), Quaternary extension in southern Tibet: Field observations and tectonic implications, J. Geophys. Res., 91, 13,803-13,872, doi:10.1029/ JB091iB14p13803.

Barth, N. C., B. R. Hacker, G. G. E. Seward, E. O. Walsh, D. Young, and S. Johnston (2010), Strain within the ultrahigh-pressure western gneiss region of Norway recorded by quartz CPOs, in Continental Tectonics and Mountain Building: The Legacy of Peach and Horne, edited by R. D. Law et al., Geol. Soc. Spec. Publ., 335, 663-685, doi:10.1144/ SP335.27

Beaumont, C., R. A. Jamieson, M. H. Nguyen, and B. Lee (2001), Himalayan tectonics explained by extrusion of a low-viscosity crustal channel coupled to focused surface denudation, Nature, 414, 738-742, doi:10.1038/414738

Beaumont, C., R. A. Jamieson, M. H. Nguyen, and S. Medvedev (2004), Crustal channel flows: 1 . Numerical models with applications to the tectonics of the Himalayan-Tibetan orogen, J. Geophys. Res., 109, B06406, doi:10.1029/2003JB002809.

Beaumont, C., M. H. Nguyen, R. A. Jamieson, and S. Ellis (2006), Crustal flow modes in large hot orogens, in Channel Flow, Ductile Extrusion and Exhumation in Continental Collision Zones, edited by R. D. Law et al., Geol. Soc. Spec. Publ., 268, 91-145.

Brookfield, M. E. (1993), The Himalayan passive margin from Precambrian to Cretaceous, Sediment. Geol., 84,1-35, doi:10.1016/0037-0738(93) 90042-4.

Burchfiel, B. C., and L. H. Royden (1985), North-south extension within the convergent Himalayan region, Geology, 13, 679-682, doi:10.1130/0091-7613 (1985) $13<679$ :NEWTCH $>2.0 . \mathrm{CO} ; 2$

Burchfiel, B. C., C. Zhiliang, K. V. Hodges, L. H. Uping, D. Changrong, and X. Jiene (1992), The south Tibetan detachment system, Himalayan orogen: Extension contemporaneous with and parallel to shortening in a collisional mountain belt, Spec. Pap. Geol. Soc. Am., 269, 41 pp.

Burg, J. P., and J. M. Chen (1984), Tectonics and structural zonation of southern Tibet, China, Nature, 311, 219-223, doi:10.1038/311219a0.

Burg, J. P., M. Brunel, D. Gapais, G. M. Chen, and G. H. Liu (1984), Deformation of leucogranites of the crystalline Main Central sheet in southern Tibet (China), J. Struct. Geol., 6, 535-542, doi:10.1016/0191-8141 (84)90063-4

Burkhard, M. (1993), Calcite twins, their geometry, appearance and significance as stress-strain markers and indicators of tectonic regime: A review, J. Struct. Geol., 15, 351-368, doi:10.1016/01918141(93)90132-T.

Carosi, R., C. Montomoli, D. Rubatto, and D. Visona (2006), Normal-sense shear zones in the core of the Higher Himalayan Crystallines (Bhutan Himalaya): Evidence for extrusion?, in Channel Flow, Ductile Extrusion and Exhumation in Continental
Collision Zones, edited by R. D. Law et al., Geol. Soc. Spec. Publ., 268, 425-444.

Carosi, R., C. Montomoli, and D. Visona (2007), A structural transect in the lower Dolpo: Insights on the tectonic evolution of western Nepal, J. Asian Earth Sci., 29, 407-423, doi:10.1016/j. jseaes.2006.05.001

Chen, Z., Y. Liu, K. V. Hodges, B. C. Burchfiel, L. H. Royden, and C. Deng (1990), The Kangmar Dome: A metamorphic core complex in southern Xizang (Tibet), Science, 250, 1552-1556, doi:10.1126/science. 250.4987.1552.

Copley, A., J.-P. Avouac, and J.-Y. Royer (2010), IndiaAsia collision and the Cenozoic slowdown of the Indian plate: Implications for the forces driving plate motions, J. Geophys. Res., 115, B03410, doi:10.1029/2009JB006634.

Cottle, J., M. P. Searle, M. Horstwood, and D. J. Waters (2009), Timing of midcrustal metamorphism, melting, and deformation in the Mount Everest region of southern Tibet revealed by $\mathrm{U}(-\mathrm{Th})-\mathrm{Pb}$ geochronology, J. Geol., 117, 643-664, doi:10.1086/605994.

Dewane, T. J., D. F. Stockli, C. Hager, M. Taylor, L. Ding, J. Lee, and S. Wallis (2006), Timing of Cenozoic E-W extension in the Tangra Yum CoKung Co Rift, south-central Tibet, Eos Trans. $A G U, 87(52)$, Fall Meet. Suppl., Abstract T34C-04.

Ferrill, D. A., P. A. Morris, M. A. Evans, M. Burkhard, R. H. Groshong Jr., and C. M. Onasch (2004), Calcite twin morphology: A low temperature deformation geothermometer, J. Struct. Geol., 26, 1521-1529, doi:10.1016/j.jsg.2003.11.028.

Gaetani, M., and E. Garzanti (1991), Multicyclic history of the northern India continental margin (northwestern Himalaya), Am. Assoc. Pet. Geol. Bull., 75, $1427-1446$.

Garzanti, E. (1999), Stratigraphy and sedimentary history of the Nepal Tethys Himalaya passive margin, J. Asian Earth Sci., 17, 805-827, doi:10.1016/ S1367-9120(99)00017-6.

Godin, L., T. P. Gleeson, M. P. Searle, T. D. Ullrich, and R. R. Parrish (2006), Locking of southward extrusion in favour of rapid crustal-scale buckling of the Greater Himalayan sequence, Nar Valley, central Nepal, in Channel Flow, Ductile Extrusion and Exhumation in Continental Collision Zones, edited by R. D. Law et al., Geol. Soc. Spec. Publ., 268, 269-292.

Grasemann, B., H. Fritz, and J. C. Vannay (1999), Quantitative kinematic flow analysis from the Main Central Thrust Zone (NW-Himalaya, India): Implications for a decelerating strain path and the extrusion of orogenic wedges, J. Struct. Geol., 21, 837-853, doi:10.1016/S0191-8141(99)00077-2.

Grujic, D. (2006), Channel flow and continental collision tectonics: An overview, in Channel Flow, Ductile Extrusion and Exhumation in Continental Collision Zones, edited by R. D. Law et al., Geol. Soc. Spec. Publ., 268, 25-37.

Grujic, D., C. Martin, C. Davidson, L. S. Hollister, R. Kuendig, T. L. Pavlis, and S. M. Schmid (1996), Ductile extrusion of the Higher Himalayan Crystalline in Bhutan: Evidence from quartz microfabrics, Tectonophysics, 260, 21-43, doi:10.1016/00401951(96)00074-1

Grujic, D., L. S. Hollister, and R. R. Parrish (2002), Himalayan metamorphic sequence as an orogenic channel: Insight from Bhutan, Earth Planet. Sci. Lett., 198, 177-191, doi:10.1016/S0012-821X(02) 00482-X.

Hager, C., D. F. Stockli, T. J. Dewane, and L. Ding (2006), Episodic Mio-Pliocene rifting in southcentral Tibet. Thermochronometric constraints from the Xainza rift, Eos Trans. $A G U, 87(52)$, Fall Meet Suppl., Abstract T34C-02.

Hauck, M. L., K. D. Nelson, L. D. Brown, W. J. Zhao, and A. R. Ross (1998), Crustal structure of the Himalayan orogen at similar to 90 degrees east longitude from Project INDEPTH deep reflection profiles, Tectonics, 17, 481-500, doi:10.1029/ 98TC01314.

Hayden, H. H. (1912), The geology of the provinces of Tsan and Ü in central Tibet, Mem. Geol. Surv. India, 36, 122-198.

Heard, H. C., and N. L. Carter (1968), Experimentally induced 'natural' intragranular flow in quartz and quartzite, Am. J. Sci., 266, 1-42, doi:10.2475/ ajs.266.1.1.

Hirth, G., and J. Tullis (1992), Dislocation creep regimes in quartz aggregates, J. Struct. Geol., 14 , 145-159, doi:10.1016/0191-8141(92)90053-Y.

Hodges, K. V., R. R. Parrish, T. B. Housh, D. R. Lux, B. C. Burchfiel, L. H. Royden, and Z. Chen (1992), Simultaneous Miocene extension and shortening in the Himalayan orogen, Science, 258, 1466-1470, doi:10.1126/science. 258.5087 .1466

Hodges, K. V., R. R. Parrish, and M. P. Searle (1996), Tectonic evolution of the central Annapurna Range, Nepalese Himalayas, Tectonics, 15, 1264-1291, doi:10.1029/96TC01791.

Hodges, K. V., J. M. Hurtado, and K. X. Whipple (2001), Southward extrusion of Tibetan crust and its effect on Himalayan tectonics, Tectonics, 20, 799-809, doi:10.1029/2001TC001281.

Jessup, M. J., R. D. Law, M. P. Searle, and M. S. Hubbard (2006), Structural evolution and vorticity of flow during extrusion and exhumation of the Greater Himalayan Slab, Mount Everest Massif, Tibet/Nepal: Implications for orogen-scale flow partitioning, in Channel Flow, Ductile Extrusion and Exhumation in Continental Collision Zones, edited by R. D. Law et al., Geol. Soc. Spec. Publ., 268, 379-413.

Jessup, M. J., R. D. Law, and C. Frassi (2007), The rigid grain net (RGN): An alternative method for estimating mean kinematic vorticity number $\left(W_{\mathrm{m}}\right), J$. Struct Geol., 29, 411-421, doi:10.1016/j.jsg.2006.11.003.

Johnson, S. E., H. J. Lenferink, N. A. Price, J. H. Marsh, P. O. Koons, D. P. West Jr., and R. Beane (2009), Clast-based kinematic vorticity gauges: The effects of slip at matrix/clast interfaces, J. Struct. Geol., 31, 1322-1339, doi:10.1016/j. jsg.2009.07.008.

Kawakami, T., M. Aoya, S. R. Wallis, J. Lee, K. Terada, Y. Wang, and M. Heizler (2007), Contact metamorphism in the Malashan Dome, north Himalayan gneiss domes, southern Tibet: An example of shallow extensional tectonics in the Tethys Himalaya, J. Metamorph. Geol., 25, 831-853, doi:10.1111/ j.1525-1314.2007.00731.x.

Kellett, D. A., D. Grujic, and S. Erdmann (2009), Miocene structural reorganization of the south Tibetan detachment, eastern Himalaya: Implications for continental collision, Lithosphere, 1, 259-281, doi:10.1130/L56.1

Koch, P. S., J. M. Christie, A. Ord, and R. P. George (1989), Effect of water on the rheology of experimentally deformed quartzite, J. Geophys. Res., 94, 13,975-13,996, doi:10.1029/JB094iB10p13975.

Langille, J., J. Lee, B. Hacker, and G. Seward (2010), Middle crustal ductile deformation patterns in southern Tibet: Insights from vorticity studies in Mabja Dome, J. Struct. Geol., 32, 70-85, doi:10.1016/j.jsg.2009.08.009.

Larson, K. P., and L. Godin (2009), Kinematics of the Greater Himalayan sequence, Dhaulagiri Himal: Implications for the structural framework of centra 
Nepal, J. Geol. Soc., 166, 25-43, doi:10.1144/001676492007-180

Law, R. D., M. P. Searle, and R. L. Simpson (2004), Strain, deformation temperatures and vorticity of flow at the top of the Greater Himalayan Slab, Everest Massif, Tibet, J. Geol. Soc. London, 161, 305-320, doi:10.1144/0016-764903-047.

Lee, J., and M. J. Whitehouse (2007), Onset of midcrustal extensional flow in southern Tibet: Evidence from $\mathrm{U} / \mathrm{Pb}$ zircon ages, Geology, 35, 45-48, doi: $10.1130 / \mathrm{G} 22842 \mathrm{~A} .1$.

Lee, J., W. S. Dinklage, B. R. Hacker, Y. Wang, P. B. Gans, A. Calvert, J. Wan, W. Chen, A. Blythe, and W. McClelland (2000), Evolution of the Kangmar Dome, southern Tibet: Structural, petrologic, and thermochronologic constraints, Tectonics, 19, 872896, doi:10.1029/1999TC001147.

Lee, J., J. L. Wan, W. S. Dinklage, and Y. Wang (2002), Geology of Kangmar Dome, southern Tibet, with explanatory notes, Map Chart Ser. MCH090, 8 pp., Geol. Soc. of Am., Boulder, Colo.

Lee, J., B. R. Hacker, and Y. Wang (2004), Evolution of North Himalayan gneiss domes: Structural and metamorphic studies in Mabja Dome, southern Tibet, J. Struct. Geol., 26, 2297-2316, doi:10.1016/j. jsg.2004.02.013.

Lee, J., W. McClelland, Y. Wang, A. Blythe, and M. McWilliams (2006), Oligocene-Miocene mid-crustal flow in southern Tibet: Geochronology of Mabja Dome, in Channel Flow, Ductile Extrusion and Exhumation in Continental Collision Zones, edited by R. D. Law et al., Geol. Soc. Spec. Publ., 268, 445-469.

Liao, Q., D. Li, L. Lu, Y. Yuan, and L. Chu (2008), Paleoproterozoic granitic gneisses of the Dinggye and LhagoiKangri areas from the higher and northern Himalaya, Tibet: Geochronology and implications, Sci. China, Ser. E, Earth Sci., 51, 240-248.

Liu, G., and G. Einsele (1994), Sedimentary history of the Tethyan basin in the Tibetan Himalayas, Geol. Rundsch., 83, 32-61, doi:10.1007/BF00211893.

Mahéo, G., P. H. Leloup, F. Valli, R. Lacassin, N. Arnaud, J.-L. Paquette, A. Fernandez, L. Haibing, K. A. Farley, and P. Tapponnier (2007), Post $4 \mathrm{Ma}$ initiation of normal faulting in southern Tibet: Constraints from the Kung Co half graben, Earth Planet. Sci. Lett., 256, 233-243, doi:10.1016/j. eps1.2007.01.029.

Mainprice, D., J.-L. Bouchez, P. Blumenfeld, and J. M. Tubià (1986), Dominant $c$ slip in naturally deformed quartz: Implications for dramatic plastic softening at high temperature, Geology, 14, 819822, doi: 10.1130/0091-7613(1986)14<819: DCSIND>2.0.CO;2.

Makovsky, Y., S. L. Klemperer, L. Ratschbacher, L. D. Brown, M. Li, W. Zhao, and F. Meng (1996), INDEPTH wide-angle reflection observation of $P$-wave-to- $S$-wave conversion from crustal bright spots in Tibet, Science, 274, 1690-1691, doi:10.1126/science.274.5293.1690.

Molnar, P., and J. M. Stock (2009), Slowing of India's convergence with Eurasia since $20 \mathrm{Ma}$ and its implications for Tibetan mantle dynamics, Tectonics, 28, TC3001, doi:10.1029/2008TC002271.

Murphy, M. A., and T. M. Harrison (1999), Relationship between leucogranites and the Qomolangma detachment in the Rongbuk Valley, south Tibet, Geology, 27, 831-834, doi:10.1130/0091-7613 (1999)027<0831:RBLATQ >2.3.CO;2.

Nelson, K. D., et al. (1996), Partially molten middle crust beneath southern Tibet: Synthesis of project
INDEPTH results, Science, 274, 1684-1688, doi:10.1126/science.274.5293.1684

Passchier, C. W. (1987), Stable positions of rigid objects in non-coaxial flow: A study in vorticity analysis, J. Struct. Geol., 9, 679-690, doi:10.1016/0191-8141(87)90152-0.

Passchier, C. W., and R. A. J. Trouw (2005), Microtectonics, 2nd ed., 366 pp., Springer, Berlin.

Pfiffner, O. A., and J. G. Ramsay (1982), Constraints on geological strain rates: Arguments from finite strain states of naturally deformed rocks, J. Geophys. Res., 87, 311-321, doi:10.1029/JB087iB01p00311.

Quigley, M., L. Yu, X. Liu, C. J. L. Wilson, M. Sandiford, and D. Phillips (2006), ${ }^{40} \mathrm{Ar} /{ }_{39} \mathrm{Ar}$ thermochronology of the Kampa Dome, southern Tibet: Implications for tectonic evolution of the North Himalayan gneiss domes, Tectonophysics, 421, 269-297, doi:10.1016/j.tecto.2006.05.002.

Quigley, M. C., L. Yu, C. Gregory, A. Corvino, M. Sandiford, C. J. L. Wilson, and X. Liu (2008), $\mathrm{U}-\mathrm{Pb}$ SHRIMP zircon geochronology and $T-t-d$ history of the Kampa Dome, southern Tibet, Tectonophysics, 446, 97-113, doi:10.1016/j.tecto.2007. 11.004.

Ramsay, J. G., and M. I. Huber (1987), The Techniques of Modern Structural Geology, vol. 2, Folds and Fractures, 700 pp., Academic, London.

Ratschbacher, L., W. Frisch, G. Liu, and C. Chen (1994), Distributed deformation in southern and western Tibet during and after the India-Asia collision, J. Geophys. Res., 99, 19,917-19,945, doi:10.1029/94JB00932.

Rowley, D. B., and B. S. Currie (2006), Paleo-altimetry of the late Eocene to Miocene Lunpola basin, central Tibet, Nature, 439, 677-681, doi:10.1038 nature04506.

Royden, L. H., and B. C. Burchfiel (1987), Thinskinned north-south extension within the convergent Himalayan region: Gravitational collapse of a Miocene topographic front, in Continental Extensional Tectonics, edited by M. P. Coward et al., Geol. Soc. Spec. Publ., 28, 611-619.

Searle, M. P., R. D. Law, R. R. Parrish, and D. J. Waters (2003), The structural geometry, metamorphic and magmatic evolution of the Everest massif, High Himalaya of Nepal-South Tibet, J. Geol. Soc., 160, 345-366, doi:10.1144/0016-764902-126.

Simpson, R. L., R. R. Parrish, M. P. Searle, and D. J. Waters (2000), Two episodes of monazite crystallization during metamorphism and crustal melting in the Everest region of the Nepalese Himalaya, Geology, 28, 403-406, doi:10.1130/0091-7613(2000)28<403 TEOMCD $>2.0 . \mathrm{CO} ; 2$.

Spear, F. S. (1993), Metamorphic Phase Equilibria and Pressure-Temperature-Time Paths, 799 pp., Mineral. Soc. of Am., Washington, D. C

Stipp, M., H. Stünitz, R. Heilbronner, and S. M. Schmid (2002a), The eastern Tonale fault zone: A 'natural laboratory' for crystal plastic deformation of quartz over a temperature range from 250 to $700^{\circ} \mathrm{C}$, J. Struct. Geol., 24, 1861-1884, doi:10.1016/ S0191-8141(02)00035-4

Stipp, M., H. Stünitz, R. Heilbronner, and S. M. Schmid (2002b), Dynamic recrystallization of quartz: Correlation between natural and experimental conditions, in Deformation Mechanisms, Rheology and Tectonics: Current Status and Future Perspectives, edited by S. De Meer et al., Geol. Soc. Spec. Publ., 200 171-190.

Stockli, D. F., M. Taylor, A. Yin, T. M. Harrison, J. D'Andrea, D. Lin, and P. Kapp (2002), Lat Miocene-Pliocene inception of $\mathrm{E}-\mathrm{W}$ extension in
Tibet as evidenced by apatite (U-Th)/He data, Geol. Soc. Am. Abstr. Programs, 34, 411

Turcotte, D., and G. Schubert (2002), Geodynamics, 456 pp., Cambridge Univ. Press, New York.

Vance, D., and N. Harris (1999), The timing of prograde metamorphism in the Zanskar Himalaya, Geology, 27, 395-398, doi:10.1130/0091-7613(1999) 027<0395:TOPMIT $>2.3 . \mathrm{CO} ; 2$

Vannay, J.-C., and B. Grasemann (2001), Himalayan inverted metamorphism and syn-convergence extension as a consequence of a general shear extrusion, Geol. Mag., 138, 253-276, doi:10.1017/ S0016756801005313.

Walker, J. D., M. W. Martin, S. A. Bowring, M. P. Searle, D. J. Waters, and K. V. Hodges (1999), Metamorphism, melting, and extension: Age constraints from the High Himalayan slab, S.E.Zanskar and N.W. Lahoul, J. Geol., 107, 473-495, doi: $10.1086 / 314360$.

Wallis, S. R. (1992), Vorticity analysis in a metachert from the Sanbagawa Belt, SW Japan, J. Struct. Geol., 14, 271-280, doi:10.1016/0191-8141(92) 90085-B.

Wallis, S. R. (1995), Vorticity analysis and recognition of ductile extension in the Sanbagawa belt, SW Japan, J. Struct. Geol., 17, 1077-1093, doi:10.1016/ 0191-8141(95)00005-X.

Wallis, S. R., J. P. Platt, and S. D. Knott (1993), Recognition of syn-convergence extension in accretionary wedges with examples from the Calabrian Arc and the Eastern Alps, Am. J. Sci., 293, 463-494, doi:10.2475/ajs.293.5.463.

White, F. M. (1974), Viscous Fluid Flow, 725 pp., McGraw-Hill, New York.

Williams, P. F., D. Jiang, and S. Lin (2006), Interpretation of deformation fabrics of infrastructure zone rocks in the context of channel flow and other models, in Channel Flow, Ductile Extrusion and Exhumation in Continental Collision Zones, edited by R. D. Law et al., Geol. Soc. Spec. Publ., 268, 221-235.

Xypolias, P. (2009), Some new aspects of kinematic vorticity analysis in naturally deformed quartzites, J. Struct. Geol., 31, 3-10, doi:10.1016/j. jsg.2008.09.009

Zhang, H., N. Harris, R. R. Parrish, S. Kelley, L. Zhang, N. Rogers, T. Argles, and J. King (2004), Causes and consequences of protracted melting of the mid-crust exposed in the north Himalayan antiform, Earth Planet. Sci. Lett., 228, 195-212, doi:10.1016/ j.epsl.2004.09.031.

Zhang, Q., Y. S. Zhou, D. Z. Li, and H. R. Wu (1986), Principal features of the gneissic dome and its peripheral metamorphic zones in Kangma of Xizang, China, Sci. Geol. Sin., 2, 125-133.

Zhou, Y. S., Q. Zhang, C. W. Jin, and H. J. Mei (1981) Magmatism and Metamorphism in Xizang (Tibet). The Series of the Scientific Expedition to QinghaiXizang, 359 pp., Sci. Press, Beijing.

B. R. Hacker and G. Seward, Department of Earth Science, University of California, Bldg. 526, Santa Barbara, CA 93106-9630, USA. (hacker@geol.ucsb. edu; seward@geol.ucsb.edu)

J. Lee, Department of Geological Sciences, Central Washington University, 400 E. 8th Ave., Ellensburg, WA 98926, USA. (jeff@geology.cwu.edu)

T. Wagner, Cabot Oil and Gas, Pittsburgh, Penn Center West, Ste. 401, Pittsburgh, PA 15276, USA. (tom.wagner@cabotog.com) 